\title{
Supplemental Table 57
}

\section{DHR Site List Lean $p<1 e-05$}

\begin{tabular}{|c|c|c|c|c|c|c|c|c|c|c|c|}
\hline DHR Name & Chr & Start & Stop & Length & \begin{tabular}{|l} 
\# Sig \\
Win
\end{tabular} & $\min P$ & $\operatorname{maxLFC}$ & CpG \# & $\begin{array}{c}\text { CpG } \\
\text { Density }\end{array}$ & Gene Annotation & Gene Category \\
\hline DHR1:175001 & 1 & 175001 & 176000 & 1000 & 1 & $8.97 \mathrm{E}-07$ & 0.6904805 & 9 & 0.9 & & \\
\hline DHR1:14997001 & 1 & 14997001 & 14998000 & 1000 & 1 & $3.25 \mathrm{E}-06$ & 0.9138199 & 16 & 1.6 & & \\
\hline DHR1:24432001 & 1 & 24432001 & 24433000 & 1000 & 1 & $5.01 \mathrm{E}-08$ & 0.8983316 & 4 & 0.4 & & \\
\hline DHR1:24686001 & 1 & 24686001 & 24687000 & 1000 & 1 & $9.89 \mathrm{E}-06$ & 0.9158629 & 9 & 0.9 & & \\
\hline DHR1:25938001 & 1 & 25938001 & 25940000 & 2000 & 1 & $9.89 \mathrm{E}-07$ & 1.3368908 & 67 & 3.35 & & \\
\hline DHR1:27676001 & 1 & 27676001 & 27677000 & 1000 & 1 & $1.23 \mathrm{E}-06$ & -1.4474879 & 2 & 0.2 & Nkain2 & Transport \\
\hline DHR1:29254001 & 1 & 29254001 & 29255000 & 1000 & 1 & $9.65 \mathrm{E}-06$ & 0.910554 & 13 & 1.3 & Ncoa7 & \\
\hline DHR1:43666001 & 1 & 43666001 & 43667000 & 1000 & 1 & $9.66 \mathrm{E}-06$ & -1.3168746 & 2 & 0.2 & Oprm1;AABR07001433.1 & Receptor \\
\hline DHR1:43852001 & 1 & 43852001 & 43854000 & 2000 & 1 & $6.47 \mathrm{E}-06$ & 0.9428896 & 14 & 0.7 & Cnksr3 & Signaling \\
\hline DHR1:53901001 & 1 & 53901001 & 53904000 & 3000 & 1 & $4.41 \mathrm{E}-08$ & 0.9841509 & 37 & 1.233 & AABR07001592.2 & \\
\hline DHR1:54499001 & 1 & 54499001 & 54501000 & 2000 & 1 & 1.47E-06 & -2.1587265 & 5 & 0.25 & AABR07001640.1 & \\
\hline DHR1:60582001 & 1 & 60582001 & 60584000 & 2000 & 1 & 9.45E-06 & -1.5319049 & 6 & 0.3 & & \\
\hline DHR1:62113001 & 1 & 62113001 & 62114000 & 1000 & 1 & 5.17E-06 & -1.3386302 & 16 & 1.6 & AABR07001923.1 & \\
\hline DHR1:67337001 & 1 & 67337001 & 67338000 & 1000 & 1 & $1.00 \mathrm{E}-06$ & 1.1143191 & 31 & 3.1 & LOC100362054 & \\
\hline DHR1:75369001 & 1 & 75369001 & 75371000 & 2000 & 1 & $1.48 \mathrm{E}-07$ & -1.9369636 & 11 & 0.55 & Lig1 & Transcription \\
\hline DHR1:78823001 & 1 & 78823001 & 78825000 & 2000 & 1 & $8.46 \mathrm{E}-06$ & 1.0513482 & 17 & 0.85 & Gng8;Ptgir & Signaling;Receptor \\
\hline DHR1:80850001 & 1 & 80850001 & 80852000 & 2000 & 1 & $4.25 \mathrm{E}-06$ & 1.117747 & 44 & 2.2 & $\operatorname{lgsf23}$ & \\
\hline DHR1:83491001 & 1 & 83491001 & 83493000 & 2000 & 1 & $2.95 \mathrm{E}-06$ & 0.9750265 & 12 & 0.6 & & \\
\hline DHR1:85929001 & 1 & 85929001 & 85931000 & 2000 & 1 & $4.66 \mathrm{E}-06$ & -1.5463337 & 7 & 0.35 & & \\
\hline DHR1:92137001 & 1 & 92137001 & 92142000 & 5000 & 1 & $3.71 \mathrm{E}-09$ & 1.1987578 & 81 & 1.62 & & \\
\hline DHR1:98287001 & 1 & 98287001 & 98288000 & 1000 & 1 & $2.70 \mathrm{E}-06$ & -1.353102 & 6 & 0.6 & & \\
\hline DHR1:101485001 & 1 & 101485001 & 101486000 & 1000 & 1 & $9.02 \mathrm{E}-06$ & 1.0138652 & 8 & 0.8 & Dhdh;Tulp2;Nucb1 & Metabolism;Transcription \\
\hline DHR1:102844001 & 1 & 102844001 & 102846000 & 2000 & 1 & $5.34 \mathrm{E}-09$ & 1.20797 & 40 & 2 & Saa4;Gtf2h1 & Transport;Transcription \\
\hline DHR1:113743001 & 1 & 113743001 & 113746000 & 3000 & 1 & $3.84 \mathrm{E}-07$ & -1.5993622 & 11 & 0.367 & AABR07003510.1 & \\
\hline DHR1:116595001 & 1 & 116595001 & 116598000 & 3000 & 1 & $3.40 \mathrm{E}-06$ & -1.3312776 & 5 & 0.167 & Ube3a & Metabolism \\
\hline DHR1:122543001 & 1 & 122543001 & 122544000 & 1000 & 1 & $3.08 \mathrm{E}-06$ & -1.3787446 & 3 & 0.3 & & \\
\hline DHR1:128279001 & 1 & 128279001 & 128280000 & 1000 & 1 & $1.18 \mathrm{E}-06$ & -1.2528844 & 7 & 0.7 & Mef2a & Transcription \\
\hline DHR1:140778001 & 1 & 140778001 & 140780000 & 2000 & 1 & $6.18 \mathrm{E}-06$ & 0.9869065 & 19 & 0.95 & Acan & Extracellular Matrix \\
\hline DHR1:150720001 & 1 & 150720001 & 150721000 & 1000 & 1 & $4.72 \mathrm{E}-06$ & -1.0839969 & 1 & 0.1 & & \\
\hline DHR1:155585001 & 1 & 155585001 & 155586000 & 1000 & 1 & $6.87 \mathrm{E}-07$ & -1.4794752 & 7 & 0.7 & & \\
\hline DHR1:159666001 & 1 & 159666001 & 159668000 & 2000 & 1 & $9.35 \mathrm{E}-07$ & -1.3413719 & 8 & 0.4 & & \\
\hline DHR1:165843001 & 1 & 165843001 & 165846000 & 3000 & 1 & $3.44 \mathrm{E}-06$ & -0.7889515 & 26 & 0.867 & Fam168a & \\
\hline DHR1:168397001 & 1 & 168397001 & 168399000 & 2000 & 1 & $7.11 \mathrm{E}-06$ & -1.1163191 & 7 & 0.35 & Olr87;OIr88 & Receptor \\
\hline DHR1:189379001 & 1 & 189379001 & 189381000 & 2000 & 1 & 7.77E-06 & -1.1863235 & 16 & 0.8 & Acsm2 & Metabolism \\
\hline DHR1:198641001 & 1 & 198641001 & 198645000 & 4000 & 1 & $4.80 \mathrm{E}-06$ & 1.060546 & 59 & 1.475 & Cd2bp2;Tbc1d10b & Transcription;Signaling \\
\hline DHR1:200296001 & 1 & 200296001 & 200297000 & 1000 & 1 & $2.85 \mathrm{E}-07$ & 1.4137926 & 8 & 0.8 & & \\
\hline DHR1:202591001 & 1 & 202591001 & 202592000 & 1000 & 1 & $6.98 \mathrm{E}-06$ & -1.5939303 & 1 & 0.1 & & \\
\hline DHR1:205758001 & 1 & 205758001 & 205761000 & 3000 & 1 & $2.41 \mathrm{E}-06$ & 1.0909489 & 37 & 1.233 & Mmp21;Uros & Protease;Metabolism \\
\hline DHR1:209776001 & 1 & 209776001 & 209777000 & 1000 & 1 & $6.49 \mathrm{E}-06$ & 0.9177143 & 10 & 1 & GIrx3 & Electron Transport \\
\hline DHR1:217617001 & 1 & 217617001 & 217618000 & 1000 & 1 & $3.14 \mathrm{E}-06$ & 1.3013129 & 24 & 2.4 & Cttn & Cytoskeleton \\
\hline DHR1:217941001 & 1 & 217941001 & 217943000 & 2000 & 1 & $7.02 \mathrm{E}-06$ & 1.0681172 & 23 & 1.15 & AC095937.1 & \\
\hline DHR1:224091001 & 1 & 224091001 & 224093000 & 2000 & 1 & $1.00 \mathrm{E}-07$ & -1.2915663 & 7 & 0.35 & & \\
\hline DHR1:229864001 & 1 & 229864001 & 229866000 & 2000 & 1 & $1.41 \mathrm{E}-06$ & -1.2653695 & 3 & 0.15 & & \\
\hline DHR1:234073001 & 1 & 234073001 & 234076000 & 3000 & 1 & $5.28 \mathrm{E}-06$ & -1.3876922 & 22 & 0.733 & & \\
\hline DHR1:237332001 & 1 & 237332001 & 237334000 & 2000 & 1 & $2.30 \mathrm{E}-06$ & -1.1947816 & 11 & 0.55 & & \\
\hline DHR1:238442001 & 1 & 238442001 & 238443000 & 1000 & 1 & $4.06 \mathrm{E}-08$ & -1.6169347 & 9 & 0.9 & Tmc1 & \\
\hline DHR1:243636001 & 1 & 243636001 & 243639000 & 3000 & 1 & 4.97E-07 & 1.0494862 & 59 & 1.967 & & \\
\hline DHR1:262213001 & 1 & 262213001 & 262214000 & 1000 & 1 & $7.22 \mathrm{E}-06$ & -0.9534454 & 3 & 0.3 & & \\
\hline DHR1:263399001 & 1 & 263399001 & 263400000 & 1000 & 1 & $4.62 \mathrm{E}-06$ & 0.8980702 & 13 & 1.3 & & \\
\hline DHR1:272156001 & 1 & 272156001 & 272158000 & 2000 & 1 & 5.49E-06 & -1.5019023 & 9 & 0.45 & & \\
\hline DHR1:275288001 & 1 & 275288001 & 275289000 & 1000 & 1 & $5.90 \mathrm{E}-06$ & -1.1523391 & 3 & 0.3 & & \\
\hline DHR1:280835001 & 1 & 280835001 & 280837000 & 2000 & 1 & $2.40 \mathrm{E}-06$ & 0.8413799 & 37 & 1.85 & & \\
\hline DHR2:5226001 & 2 & 5226001 & 5228000 & 2000 & 1 & $8.39 \mathrm{E}-06$ & -1.9980382 & 5 & 0.25 & Fam172a & Unknown \\
\hline DHR2:15504001 & 2 & 15504001 & 15505000 & 1000 & 1 & $5.20 \mathrm{E}-06$ & -1.4378341 & 5 & 0.5 & & \\
\hline DHR2:34326001 & 2 & 34326001 & 34327000 & 1000 & 1 & $4.91 \mathrm{E}-06$ & 0.9632708 & 5 & 0.5 & Cenpk & Cell Cycle \\
\hline DHR2:36988001 & 2 & 36988001 & 36989000 & 1000 & 1 & $8.94 \mathrm{E}-06$ & -1.3008798 & 2 & 0.2 & & \\
\hline DHR2:42038001 & 2 & 42038001 & 42040000 & 2000 & 1 & $8.41 \mathrm{E}-06$ & -1.248826 & 8 & 0.4 & & \\
\hline DHR2:48766001 & 2 & 48766001 & 48767000 & 1000 & 1 & $7.42 \mathrm{E}-06$ & -1.0771915 & 3 & 0.3 & & \\
\hline DHR2:54301001 & 2 & 54301001 & 54303000 & 2000 & 1 & $7.87 \mathrm{E}-07$ & -1.0491576 & 10 & 0.5 & Plcxd3 & \\
\hline DHR2:55799001 & 2 & 55799001 & 55800000 & 1000 & 1 & $2.79 \mathrm{E}-06$ & -1.4941357 & 4 & 0.4 & C9 & Immune \\
\hline DHR2:61918001 & 2 & 61918001 & 61919000 & 1000 & 1 & $4.52 \mathrm{E}-06$ & -1.4192618 & 2 & 0.2 & Npr3 & Receptor \\
\hline DHR2:66656001 & 2 & 66656001 & 66658000 & 2000 & 1 & $5.61 \mathrm{E}-08$ & -1.7467087 & 6 & 0.3 & & \\
\hline DHR2:67446001 & 2 & 67446001 & 67452000 & 6000 & 1 & $4.15 \mathrm{E}-06$ & -1.5325485 & 37 & 0.617 & & \\
\hline DHR2:70350001 & 2 & 70350001 & 70353000 & 3000 & 1 & $7.00 \mathrm{E}-06$ & -1.0456407 & 19 & 0.633 & & \\
\hline
\end{tabular}




\begin{tabular}{|c|c|c|c|c|c|c|c|c|c|c|c|}
\hline DHR2:75291001 & 2 & 75291001 & 75293000 & 2000 & 1 & $6.68 \mathrm{E}-06$ & -1.5541176 & 4 & 0.2 & & \\
\hline DHR2:76067001 & 2 & 76067001 & 76077000 & 10000 & 1 & $7.66 \mathrm{E}-06$ & -1.9922643 & 44 & 0.44 & U1 & \\
\hline DHR2:90007001 & 2 & 90007001 & 90008000 & 1000 & 1 & $1.48 \mathrm{E}-06$ & -1.2578619 & 2 & 0.2 & & \\
\hline DHR2:90165001 & 2 & 90165001 & 90167000 & 2000 & 1 & $1.39 \mathrm{E}-06$ & -1.301819 & 6 & 0.3 & & \\
\hline DHR2:96943001 & 2 & 96943001 & 96945000 & 2000 & 1 & $7.18 \mathrm{E}-06$ & -1.4154469 & 6 & 0.3 & & \\
\hline DHR2:127049001 & 2 & 127049001 & 127050000 & 1000 & 1 & $2.47 \mathrm{E}-06$ & -1.1348125 & 6 & 0.6 & & \\
\hline DHR2:132813001 & 2 & 132813001 & 132814000 & 1000 & 1 & $2.52 \mathrm{E}-06$ & -1.2998916 & 4 & 0.4 & & \\
\hline DHR2:138351001 & 2 & 138351001 & 138354000 & 3000 & 1 & $6.48 \mathrm{E}-06$ & -1.5326711 & 30 & 1 & & \\
\hline DHR2:139356001 & 2 & 139356001 & 139358000 & 2000 & 1 & 3.19E-06 & -1.3053107 & 11 & 0.55 & & \\
\hline DHR2:139717001 & 2 & 139717001 & 139720000 & 3000 & 1 & $6.63 \mathrm{E}-06$ & 0.9584164 & 23 & 0.767 & & \\
\hline DHR2:146805001 & 2 & 146805001 & 146808000 & 3000 & 1 & 6.39E-06 & -1.0687496 & 13 & 0.433 & & \\
\hline DHR2:151115001 & 2 & 151115001 & 151116000 & 1000 & 1 & $4.86 \mathrm{E}-06$ & -1.4256608 & 2 & 0.2 & & \\
\hline DHR2:152205001 & 2 & \begin{tabular}{|l|}
152205001 \\
\end{tabular} & 152206000 & 1000 & 1 & $2.98 \mathrm{E}-06$ & -1.464597 & 2 & 0.2 & & \\
\hline DHR2:152947001 & 2 & 152947001 & 152948000 & 1000 & 1 & $3.74 \mathrm{E}-07$ & -1.4264093 & 3 & 0.3 & & \\
\hline DHR2:153032001 & 2 & 153032001 & 153033000 & 1000 & 1 & $4.33 \mathrm{E}-06$ & 0.9298896 & 8 & 0.8 & & \\
\hline DHR2:153101001 & 2 & 153101001 & 153103000 & 2000 & 1 & $1.77 \mathrm{E}-06$ & -1.2544595 & 12 & 0.6 & & \\
\hline DHR2:162124001 & 2 & 162124001 & 162125000 & 1000 & 1 & $8.59 \mathrm{E}-06$ & 0.9646362 & 8 & 0.8 & & \\
\hline DHR2:162362001 & 2 & 162362001 & 162363000 & 1000 & 1 & $6.00 \mathrm{E}-06$ & -1.222288 & 2 & 0.2 & & \\
\hline DHR2:168096001 & 2 & 168096001 & 168101000 & 5000 & 1 & $5.01 \mathrm{E}-06$ & -1.5360041 & 37 & 0.74 & & \\
\hline DHR2:171787001 & 2 & 171787001 & \begin{tabular}{|l|}
171790000 \\
\end{tabular} & 3000 & 1 & $7.88 \mathrm{E}-06$ & -1.1219829 & 32 & 1.067 & & \\
\hline DHR2:175835001 & 2 & 175835001 & 175837000 & 2000 & 1 & $7.51 \mathrm{E}-06$ & -1.5492739 & 8 & 0.4 & & \\
\hline DHR2:185199001 & 2 & 185199001 & 185200000 & 1000 & 1 & $1.23 \mathrm{E}-06$ & 0.9844025 & 12 & 1.2 & AABR07012092.1 & \\
\hline DHR2:187761001 & 2 & 187761001 & 187764000 & 3000 & 1 & $4.59 \mathrm{E}-07$ & 0.9153662 & 36 & 1.2 & Pmf1 & \\
\hline DHR2:189839001 & 2 & 189839001 & 189841000 & 2000 & 1 & $9.57 \mathrm{E}-06$ & 1.1841249 & 22 & 1.1 & Npr1 & Receptor \\
\hline DHR2:190852001 & 2 & 190852001 & 190854000 & 2000 & 1 & $1.01 \mathrm{E}-06$ & -2.122228 & 7 & 0.35 & & \\
\hline DHR2:200747001 & 2 & 200747001 & 200748000 & 1000 & 1 & $5.37 \mathrm{E}-06$ & -1.3200323 & 2 & 0.2 & $\mathrm{Hsd} 3 \mathrm{~b} 3$ & Metabolism \\
\hline DHR2:204713001 & 2 & 204713001 & 204714000 & 1000 & 1 & $5.48 \mathrm{E}-07$ & -0.7965941 & 10 & 1 & & \\
\hline DHR2:214605001 & 2 & 214605001 & 214606000 & 1000 & 1 & $1.26 \mathrm{E}-06$ & -1.981346 & 4 & 0.4 & & \\
\hline DHR2:215412001 & 2 & 215412001 & 215416000 & 4000 & 1 & $3.99 \mathrm{E}-06$ & -1.5679011 & 19 & 0.475 & & \\
\hline DHR2:216218001 & 2 & 216218001 & 216220000 & 2000 & 1 & $4.30 \mathrm{E}-06$ & -1.1157494 & 5 & 0.25 & & \\
\hline DHR2:224695001 & 2 & 224695001 & 224696000 & 1000 & 1 & $6.99 \mathrm{E}-06$ & -1.0087293 & 2 & 0.2 & & \\
\hline DHR2:225941001 & 2 & 225941001 & 225944000 & 3000 & 1 & $2.03 \mathrm{E}-06$ & 0.9580321 & 41 & 1.367 & AABR07013167.1 & \\
\hline DHR2:226965001 & 2 & 226965001 & 226966000 & 1000 & 1 & $6.43 \mathrm{E}-07$ & -1.4421797 & 8 & 0.8 & Pde5a & Metabolism \\
\hline DHR2:228140001 & 2 & 228140001 & 228142000 & 2000 & 1 & $2.31 \mathrm{E}-06$ & -1.1462279 & 11 & 0.55 & & \\
\hline DHR2:244705001 & 2 & 244705001 & 244708000 & 3000 & 1 & $4.21 \mathrm{E}-06$ & -1.3446314 & 22 & 0.733 & Stpg2 & Development \\
\hline DHR2:245964001 & 2 & 245964001 & 245965000 & 1000 & 1 & $7.64 \mathrm{E}-06$ & -1.069649 & 10 & 1 & & \\
\hline DHR2:247005001 & 2 & \begin{tabular}{|l|}
247005001 \\
\end{tabular} & 247006000 & 1000 & 1 & $2.42 \mathrm{E}-06$ & -1.1819925 & 1 & 0.1 & & \\
\hline DHR2:253349001 & 2 & 253349001 & 253350000 & 1000 & 1 & $4.16 \mathrm{E}-06$ & -1.3921133 & 4 & 0.4 & & \\
\hline DHR2:257845001 & 2 & 257845001 & 257846000 & 1000 & 1 & $7.36 \mathrm{E}-06$ & 0.914084 & 8 & 0.8 & Ak5 & Signaling \\
\hline DHR2:259581001 & 2 & \begin{tabular}{|l|}
259581001 \\
\end{tabular} & 259583000 & 2000 & 1 & $5.46 \mathrm{E}-07$ & 0.8488049 & 30 & 1.5 & St6galnac3 & Metabolism \\
\hline DHR2:265097001 & 2 & 265097001 & 265098000 & 1000 & 1 & $9.39 \mathrm{E}-06$ & -1.7996534 & 11 & 1.1 & Lrrc7 & Unknown \\
\hline DHR2:266324001 & 2 & 266324001 & 266326000 & 2000 & 1 & $1.58 \mathrm{E}-08$ & -1.4991428 & 7 & 0.35 & Wls & \\
\hline DHR3:15648001 & 3 & 15648001 & 15650000 & 2000 & 1 & $6.58 \mathrm{E}-06$ & -0.7200518 & 11 & 0.55 & & \\
\hline DHR3:18256001 & 3 & 18256001 & 18259000 & 3000 & 1 & $3.30 \mathrm{E}-06$ & -1.3884059 & 11 & 0.367 & & \\
\hline DHR3:23695001 & 3 & 23695001 & 23697000 & 2000 & 1 & $4.12 \mathrm{E}-06$ & -2.2518778 & 4 & 0.2 & & \\
\hline DHR3:23836001 & 3 & 23836001 & 23837000 & 1000 & 1 & $8.88 \mathrm{E}-08$ & -1.6033225 & 2 & 0.2 & & \\
\hline DHR3:26239001 & 3 & 26239001 & 26240000 & 1000 & 1 & 7.94E-06 & -1.4773409 & 6 & 0.6 & & \\
\hline DHR3:31784001 & 3 & 31784001 & 31786000 & 2000 & 1 & $6.28 \mathrm{E}-06$ & -1.1642903 & 10 & 0.5 & & \\
\hline DHR3:35374001 & 3 & 35374001 & 35375000 & 1000 & 1 & $3.22 \mathrm{E}-06$ & 1.1494254 & 2 & 0.2 & Lypd6b & \\
\hline DHR3:39444001 & 3 & 39444001 & 39445000 & 1000 & 1 & $4.44 \mathrm{E}-06$ & -1.0963453 & 3 & 0.3 & & \\
\hline DHR3:41168001 & 3 & 41168001 & 41169000 & 1000 & 1 & $3.58 \mathrm{E}-06$ & -1.0694453 & 11 & 1.1 & Kcnj3 & Transport \\
\hline DHR3:41578001 & 3 & 41578001 & 41579000 & 1000 & 1 & $9.68 \mathrm{E}-06$ & -1.0025208 & 7 & 0.7 & & \\
\hline DHR3:45465001 & 3 & 45465001 & 45468000 & 3000 & 1 & $9.89 \mathrm{E}-07$ & 0.8201837 & 38 & 1.267 & & \\
\hline DHR3:47897001 & 3 & 47897001 & 47898000 & 1000 & 1 & $8.28 \mathrm{E}-06$ & -1.1603891 & 2 & 0.2 & & \\
\hline DHR3:52398001 & 3 & 52398001 & 52399000 & 1000 & 1 & $3.14 \mathrm{E}-06$ & -1.4291608 & 0 & 0 & Scn1a & Metabolism \\
\hline DHR3:61689001 & 3 & 61689001 & 61691000 & 2000 & 1 & $5.42 \mathrm{E}-06$ & -1.4445172 & 29 & 1.45 & Hoxd1 & Transcription \\
\hline DHR3:69674001 & 3 & 69674001 & 69677000 & 3000 & 1 & $5.58 \mathrm{E}-06$ & -1.6254346 & 7 & 0.233 & & \\
\hline DHR3:74575001 & 3 & 74575001 & 74577000 & 2000 & 1 & $1.86 \mathrm{E}-06$ & -1.5573916 & 8 & 0.4 & Olr532;Olr533;AABR07052768.1 & Receptor \\
\hline DHR3:76341001 & 3 & 76341001 & 76342000 & 1000 & 1 & $6.37 \mathrm{E}-06$ & -1.3779149 & 4 & 0.4 & Olr613 & \\
\hline DHR3:87481001 & 3 & 87481001 & 87482000 & 1000 & 1 & $1.93 \mathrm{E}-06$ & -1.5415199 & 5 & 0.5 & & \\
\hline DHR3:87932001 & 3 & \begin{tabular}{|l|}
87932001 \\
\end{tabular} & \begin{tabular}{|l|}
87935000 \\
\end{tabular} & 3000 & 1 & $1.72 \mathrm{E}-06$ & -1.5125882 & 6 & 0.2 & & \\
\hline DHR3:100372001 & 3 & 100372001 & 100373000 & 1000 & 1 & $6.42 \mathrm{E}-06$ & -1.0019998 & 5 & 0.5 & Mettl15;Kif18a & Cytoskeleton \\
\hline DHR3:101170001 & 3 & 101170001 & 101173000 & 3000 & 1 & $8.41 \mathrm{E}-06$ & -1.1106948 & 10 & 0.333 & Ccdc34 & Transcription \\
\hline DHR3:104702001 & 3 & 104702001 & 104703000 & 1000 & 1 & $1.76 \mathrm{E}-06$ & -1.4630831 & 3 & 0.3 & & \\
\hline DHR3:109240001 & 3 & 109240001 & 109242000 & 2000 & 1 & $3.60 \mathrm{E}-06$ & -1.3747067 & 7 & 0.35 & & \\
\hline DHR3:110495001 & 3 & 110495001 & 110497000 & 2000 & 1 & $9.64 \mathrm{E}-06$ & 1.0180991 & 15 & 0.75 & Pak6;Ankrd63;Plcb2 & Signaling;Metabolism \\
\hline DHR3:115109001 & 3 & 115109001 & 115110000 & 1000 & 1 & $4.06 \mathrm{E}-07$ & -1.3358886 & 3 & 0.3 & & \\
\hline DHR3:120740001 & 3 & 120740001 & 120741000 & 1000 & 1 & $5.87 \mathrm{E}-06$ & 1.1016143 & 29 & 2.9 & $\mathrm{Bc}|2| 11$ & Apoptosis \\
\hline DHR3:124639001 & 3 & 124639001 & 124640000 & 1000 & 1 & $3.62 \mathrm{E}-06$ & 0.873528 & 10 & & SIc23a2 & Transport \\
\hline
\end{tabular}




\begin{tabular}{|c|c|c|c|c|c|c|c|c|c|c|c|}
\hline DHR3:132186001 & 3 & 132186001 & 132190000 & 4000 & 1 & $3.03 \mathrm{E}-06$ & \begin{tabular}{|c|}
-1.2878155 \\
\end{tabular} & 26 & 0.65 & & \\
\hline DHR3:133827001 & 3 & 133827001 & 133828000 & 1000 & 1 & $4.91 \mathrm{E}-06$ & -1.161826 & 1 & 0.1 & & \\
\hline DHR3:135088001 & 3 & 135088001 & 135090000 & 2000 & 1 & $3.31 \mathrm{E}-07$ & -1.3320411 & 6 & 0.3 & & \\
\hline DHR3:135529001 & 3 & 135529001 & 135531000 & 2000 & 1 & $1.62 \mathrm{E}-06$ & -1.3459493 & 11 & 0.55 & & \\
\hline DHR3:144751001 & 3 & 144751001 & \begin{tabular}{|l|}
144753000 \\
\end{tabular} & 2000 & 1 & $1.61 \mathrm{E}-07$ & -1.294795 & 8 & 0.4 & & \\
\hline DHR3:146015001 & 3 & 146015001 & 146017000 & 2000 & 1 & $2.25 \mathrm{E}-06$ & -1.086493 & 6 & 0.3 & & \\
\hline DHR3:147268001 & 3 & 147268001 & 147271000 & 3000 & 1 & $7.71 \mathrm{E}-06$ & 0.9080874 & 35 & 1.167 & Psmf1 & \\
\hline DHR3:152524001 & 3 & 152524001 & 152525000 & 1000 & 1 & $9.86 \mathrm{E}-06$ & 0.8972203 & 7 & 0.7 & & \\
\hline DHR3:153111001 & 3 & 153111001 & 153112000 & 1000 & 1 & $5.55 \mathrm{E}-06$ & 0.9443833 & 15 & 1.5 & Dsn1 & \\
\hline DHR3:156572001 & 3 & 156572001 & \begin{tabular}{|l|}
156575000 \\
\end{tabular} & 3000 & 1 & $9.02 \mathrm{E}-07$ & 0.9147701 & 52 & 1.733 & AABR07054490.1 & \\
\hline DHR3:156773001 & 3 & 156773001 & 156779000 & 6000 & 1 & $4.33 \mathrm{E}-06$ & 0.9881411 & 93 & 1.55 & Zhx3 & Transcription \\
\hline DHR3:159577001 & 3 & 159577001 & 159581000 & 4000 & 1 & $4.57 \mathrm{E}-06$ & 1.1702284 & 79 & 1.975 & Tox2 & \\
\hline DHR3:163075001 & 3 & 163075001 & 163076000 & 1000 & 1 & $1.24 \mathrm{E}-06$ & 0.9424812 & 14 & 1.4 & & \\
\hline DHR3:173919001 & 3 & 173919001 & 173920000 & 1000 & 1 & $2.21 \mathrm{E}-07$ & -1.418358 & 1 & 0.1 & Sycp2 & \\
\hline DHR4:14606001 & 4 & 14606001 & 14607000 & 1000 & 1 & $1.41 \mathrm{E}-06$ & $\begin{array}{l}-1.4942703 \\
\end{array}$ & 7 & 0.7 & & \\
\hline DHR4:15183001 & 4 & 15183001 & 15184000 & 1000 & 1 & $5.70 \mathrm{E}-06$ & -1.2446375 & 4 & 0.4 & & \\
\hline DHR4:15259001 & 4 & 15259001 & 15260000 & 1000 & 1 & $4.41 \mathrm{E}-06$ & -1.5187926 & 5 & 0.5 & & \\
\hline DHR4:15355001 & 4 & 15355001 & 15356000 & 1000 & 1 & $9.01 \mathrm{E}-06$ & -1.2627758 & 4 & 0.4 & & \\
\hline DHR4:17292001 & 4 & 17292001 & 17293000 & 1000 & 1 & $6.30 \mathrm{E}-06$ & -1.2718209 & 1 & 0.1 & Sema3e & Development \\
\hline DHR4:18086001 & 4 & 18086001 & 18087000 & 1000 & 1 & $7.64 \mathrm{E}-06$ & -1.5717795 & $3 \mid$ & 0.3 & & \\
\hline DHR4:24676001 & 4 & 24676001 & 24678000 & 2000 & 1 & $2.20 \mathrm{E}-08$ & $\begin{array}{l}-1.6095039 \\
\end{array}$ & \begin{tabular}{l|l}
5 \\
\end{tabular} & 0.25 & & \\
\hline DHR4:32596001 & 4 & 32596001 & 32597000 & 1000 & 1 & $6.39 \mathrm{E}-06$ & -1.2397285 & \begin{tabular}{l|l}
4 & \\
\end{tabular} & 0.4 & & \\
\hline DHR4:34115001 & 4 & 34115001 & 34116000 & 1000 & 1 & $2.63 \mathrm{E}-06$ & -1.4700461 & 6 & 0.6 & Col28a1 & \\
\hline DHR4:37136001 & 4 & 37136001 & 37137000 & 1000 & 1 & $2.13 \mathrm{E}-06$ & -1.4245131 & 1 & 0.1 & & \\
\hline DHR4:48445001 & 4 & 48445001 & 48446000 & 1000 & 1 & $3.25 \mathrm{E}-06$ & -1.2096672 & 10 & 1 & & \\
\hline DHR4:53712001 & 4 & 53712001 & 53715000 & 3000 & 1 & $8.46 \mathrm{E}-06$ & -1.1586998 & 18 & 0.6 & & \\
\hline DHR4:57968001 & 4 & 57968001 & 57971000 & 3000 & 1 & $1.57 \mathrm{E}-07$ & 0.9797417 & 53 & 1.767 & Cpa1;Cep41 & Protease \\
\hline DHR4:63714001 & 4 & 63714001 & 63716000 & 2000 & 1 & 5.47E-06 & -1.4186829 & 5 & 0.25 & & \\
\hline DHR4:66091001 & 4 & 66091001 & 66092000 & 1000 & 1 & $3.76 \mathrm{E}-06$ & 0.8174661 & 8 & 0.8 & Ttc26;AABR07060287.1 & \\
\hline DHR4:71477001 & 4 & 71477001 & 71478000 & 1000 & 1 & $2.56 \mathrm{E}-06$ & -0.9973202 & \begin{tabular}{l|}
7 \\
\end{tabular} & 0.7 & & \\
\hline DHR4:73880001 & 4 & 73880001 & 73882000 & 2000 & 1 & $1.78 \mathrm{E}-06$ & 0.9932371 & 58 & 2.9 & & \\
\hline DHR4:86483001 & 4 & 86483001 & 86485000 & 2000 & 1 & $1.18 \mathrm{E}-06$ & -1.3799428 & 8 & 0.4 & Pde1c & Metabolism \\
\hline DHR4:88026001 & 4 & 88026001 & 88028000 & 2000 & 1 & $5.40 \mathrm{E}-06$ & -1.3649268 & 5 & 0.25 & Vom1r80 & \\
\hline DHR4:88321001 & 4 & 88321001 & 88326000 & 5000 & 1 & $4.43 \mathrm{E}-07$ & -1.7320479 & 20 & 0.4 & Vom1r87 & Receptor \\
\hline DHR4:95977001 & 4 & 95977001 & 95978000 & 1000 & 1 & $3.57 \mathrm{E}-06$ & -1.0945255 & 2 & 0.2 & Hpgds & Metabolism \\
\hline DHR4:105364001 & 4 & 105364001 & 105366000 & 2000 & 1 & $3.65 \mathrm{E}-07$ & -1.4210526 & 10 & 0.5 & & \\
\hline DHR4:106642001 & 4 & 106642001 & 106644000 & 2000 & 1 & $8.47 \mathrm{E}-06$ & -1.2347334 & 11 & 0.55 & & \\
\hline DHR4:121224001 & 4 & 121224001 & 121226000 & 2000 & 1 & $7.98 \mathrm{E}-06$ & 1.0938707 & 34 & 1.7 & Plxna1 & Receptor \\
\hline DHR4:124453001 & 4 & 124453001 & 124454000 & 1000 & 1 & $6.01 \mathrm{E}-06$ & 0.9516077 & 8 & 0.8 & & \\
\hline DHR4:127574001 & 4 & 127574001 & 127575000 & 1000 & 1 & $7.41 \mathrm{E}-06$ & 0.7907236 & 14 & 1.4 & Suclg2 & Metabolism \\
\hline DHR4:137204001 & 4 & 137204001 & 137206000 & 2000 & 1 & $2.31 \mathrm{E}-06$ & -1.5850353 & 6 & 0.3 & & \\
\hline DHR4:146344001 & 4 & 146344001 & 146345000 & 1000 & 1 & $7.88 \mathrm{E}-07$ & 0.8799713 & 11 & 1.1 & & \\
\hline DHR4:153885001 & 4 & 153885001 & 153886000 & 1000 & 1 & $5.55 \mathrm{E}-06$ & 0.9599572 & 18 & 1.8 & Slc6a13 & Transport \\
\hline DHR4:156050001 & 4 & 156050001 & 156052000 & 2000 & 1 & $3.40 \mathrm{E}-06$ & -0.8635978 & 17 & 0.85 & Clec4a & Receptor \\
\hline DHR4:156289001 & 4 & 156289001 & 156291000 & 2000 & 1 & $2.39 \mathrm{E}-07$ & $\begin{array}{l}-1.334785 \\
\end{array}$ & 8 & 0.4 & & \\
\hline DHR4:159138001 & 4 & 159138001 & 159140000 & 2000 & 1 & $5.33 \mathrm{E}-06$ & 0.8743747 & 35 & 1.75 & & \\
\hline DHR4:161257001 & 4 & 161257001 & 161258000 & 1000 & 1 & $3.23 \mathrm{E}-07$ & \begin{tabular}{|c|}
-1.3030481 \\
\end{tabular} & 4 & 0.4 & & \\
\hline DHR4:167126001 & 4 & 167126001 & 167127000 & 1000 & 1 & $2.08 \mathrm{E}-06$ & -1.2007739 & 7 & 0.7 & Tas2r110 & \\
\hline DHR4:170485001 & 4 & 170485001 & 170486000 & 1000 & 1 & 8.93E-06 & -1.174559 & 0 & 0 & AABR07062350.1 & \\
\hline DHR4:170526001 & 4 & 170526001 & 170528000 & 2000 & 1 & $1.10 \mathrm{E}-06$ & -1.3280065 & 9 & 0.45 & Atf7ip & \\
\hline DHR4:173388001 & 4 & 173388001 & 173391000 & 3000 & 1 & $9.82 \mathrm{E}-06$ & 0.9183811 & 34 & 1.133 & & \\
\hline DHR5:15861001 & 5 & 15861001 & 15864000 & 3000 & 1 & $7.06 \mathrm{E}-07$ & -1.207469 & 8 & 0.267 & Xkr4 & Unknown \\
\hline DHR5:16391001 & 5 & 16391001 & 16393000 & 2000 & 1 & $6.81 \mathrm{E}-06$ & -1.2127757 & 19 & 0.95 & Tmem68 & Unknown \\
\hline DHR5:20462001 & 5 & 20462001 & 20463000 & 1000 & 1 & $6.21 \mathrm{E}-06$ & -1.5043242 & 4 & 0.4 & & \\
\hline DHR5:31636001 & 5 & 31636001 & 31638000 & 2000 & 1 & $4.42 \mathrm{E}-06$ & -1.2515139 & 14 & 0.7 & Mmp16 & Proteolysis \\
\hline DHR5:37032001 & 5 & 37032001 & 37034000 & 2000 & 1 & $3.30 \mathrm{E}-06$ & $\begin{array}{l}-1.168237 \\
\end{array}$ & 4 & 0.2 & & \\
\hline DHR5:38445001 & 5 & 38445001 & 38447000 & 2000 & 1 & $6.10 \mathrm{E}-07$ & -1.6678498 & 3 & 0.15 & & \\
\hline DHR5:41868001 & 5 & 41868001 & 41869000 & 1000 & 1 & $2.65 \mathrm{E}-06$ & -1.4138449 & 10 & 1 & & \\
\hline DHR5:53532001 & 5 & 53532001 & 53534000 & 2000 & 1 & $4.31 \mathrm{E}-06$ & -1.3336246 & 7 & 0.35 & & \\
\hline DHR5:62113001 & 5 & 62113001 & 62114000 & 1000 & 1 & $4.48 \mathrm{E}-06$ & 0.9092883 & 26 & 2.6 & Nans & Metabolism \\
\hline DHR5:67155001 & 5 & 67155001 & 67156000 & 1000 & 1 & 7.01E-06 & -1.6695476 & 4 & 0.4 & & \\
\hline DHR5:79925001 & 5 & 79925001 & 79926000 & 1000 & 1 & $9.96 \mathrm{E}-06$ & -0.8725637 & 2 & 0.2 & AC229945.2 & \\
\hline DHR5:82887001 & 5 & 82887001 & 82888000 & 1000 & 1 & $2.52 \mathrm{E}-06$ & -2.0099793 & 2 & 0.2 & & \\
\hline DHR5:85441001 & 5 & 85441001 & 85443000 & 2000 & 1 & $2.15 \mathrm{E}-06$ & -1.2228764 & 16 & 0.8 & & \\
\hline DHR5:93516001 & 5 & 93516001 & 93517000 & 1000 & 1 & $6.00 \mathrm{E}-07$ & -1.3655353 & 2 & 0.2 & & \\
\hline DHR5:94152001 & 5 & 94152001 & 94154000 & 2000 & 1 & $6.93 \mathrm{E}-06$ & -1.2387906 & 13 & 0.65 & & \\
\hline DHR5:97342001 & 5 & 97342001 & 97344000 & 2000 & 1 & $5.07 \mathrm{E}-06$ & -1.285579 & 7 & 0.35 & & \\
\hline DHR5:97480001 & 5 & 97480001 & 97481000 & 1000 & 1 & $7.06 \mathrm{E}-06$ & $\begin{array}{l}-1.3198459 \\
\end{array}$ & 3 & 0.3 & & \\
\hline DHR5:98415001 & 5 & 98415001 & 98416000 & 1000 & 1 & $9.33 \mathrm{E}-06$ & -1.2495885 & 6 & 0.6 & Tyrp1 & Metabolism \\
\hline
\end{tabular}




\begin{tabular}{|c|c|c|c|c|c|c|c|c|c|c|c|}
\hline DHR5:107363001 & 5 & 107363001 & 107365000 & 2000 & 1 & $3.76 \mathrm{E}-06$ & -0.8081941 & 14 & 0.7 & AABR07049134.3;LOC100912314 & Immune \\
\hline DHR5:113816001 & 5 & 113816001 & 113817000 & 1000 & 1 & $8.77 \mathrm{E}-06$ & -0.9503538 & 10 & 1 & Tek;AABR07049286.1 & Signaling \\
\hline DHR5:135977001 & 5 & 135977001 & 135978000 & 1000 & 1 & $1.11 \mathrm{E}-06$ & 1.2393865 & 33 & 3.3 & Ptch2;Btbd19 & \\
\hline DHR5:139616001 & 5 & 139616001 & 139617000 & 1000 & 1 & $1.25 \mathrm{E}-06$ & 1.1664641 & 28 & 2.8 & & \\
\hline DHR5:142000001 & 5 & 142000001 & 142002000 & 2000 & 1 & 4.93E-06 & 0.9373544 & 32 & 1.6 & & \\
\hline DHR5:155231001 & 5 & 155231001 & 155235000 & 4000 & 1 & $4.27 \mathrm{E}-06$ & 0.888901 & 89 & 2.225 & & \\
\hline DHR5:158808001 & 5 & 158808001 & 158811000 & 3000 & 1 & $4.13 \mathrm{E}-06$ & 0.9686698 & 80 & 2.667 & & \\
\hline DHR5:159900001 & 5 & 159900001 & 159901000 & 1000 & 1 & $8.92 \mathrm{E}-06$ & 1.0710416 & 23 & 2.3 & & \\
\hline DHR5:162739001 & 5 & 162739001 & 162740000 & 1000 & 1 & $1.88 \mathrm{E}-06$ & -1.0645756 & 2 & 0.2 & RGD1559644 & \\
\hline DHR5:170125001 & 5 & 170125001 & 170126000 & 1000 & 1 & $1.10 \mathrm{E}-08$ & 1.0961473 & 25 & 2.5 & & \\
\hline DHR5:172026001 & 5 & 172026001 & 172028000 & 2000 & 1 & $8.46 \mathrm{E}-06$ & 1.1137274 & 32 & 1.6 & & \\
\hline DHR6:2767001 & 6 & 2767001 & \begin{tabular}{|l|}
2768000 \\
\end{tabular} & 1000 & 1 & $1.91 \mathrm{E}-06$ & 1.1029902 & 20 & 2 & & \\
\hline DHR6:4597001 & 6 & 4597001 & 4599000 & 2000 & 1 & $3.28 \mathrm{E}-06$ & -1.1425368 & 10 & 0.5 & & \\
\hline DHR6:4979001 & 6 & 4979001 & 4981000 & 2000 & 1 & $9.20 \mathrm{E}-09$ & -1.4809202 & 7 & 0.35 & & \\
\hline DHR6:7993001 & 6 & 7993001 & 7994000 & 1000 & 1 & $3.05 \mathrm{E}-06$ & 0.926207 & 22 & 2.2 & Lrpprc & Development \\
\hline DHR6:9755001 & 6 & 9755001 & 9756000 & 1000 & 1 & 7.00E-06 & 0.9201645 & 17 & 1.7 & & \\
\hline DHR6:11392001 & 6 & 11392001 & 11395000 & 3000 & 1 & $6.94 \mathrm{E}-07$ & 1.1039657 & 57 & 1.9 & Kcnk12 & Transport \\
\hline DHR6:20816001 & 6 & 20816001 & 20817000 & 1000 & 1 & 4.63E-06 & -1.1871034 & 2 & 0.2 & & \\
\hline DHR6:29216001 & 6 & 29216001 & 29218000 & 2000 & 1 & $1.02 \mathrm{E}-06$ & 0.9500702 & 25 & 1.25 & Klhl29 & Transcription \\
\hline DHR6:38447001 & 6 & 38447001 & 38449000 & 2000 & 1 & 7.35E-06 & -1.2766377 & 9 & 0.45 & Ddx1 & Transcription \\
\hline DHR6:40005001 & 6 & 40005001 & 40008000 & 3000 & 1 & $1.91 \mathrm{E}-06$ & -1.5202297 & 14 & 0.467 & & \\
\hline DHR6:48714001 & 6 & 48714001 & 48715000 & 1000 & 1 & 2.13E-06 & 0.8891124 & 21 & 2.1 & Myt1l & Transcription \\
\hline DHR6:56874001 & 6 & 56874001 & 56875000 & 1000 & 1 & $1.50 \mathrm{E}-06$ & -1.2450602 & 6 & 0.6 & Agmo & Metabolism \\
\hline DHR6:59484001 & 6 & 59484001 & 59487000 & 3000 & 1 & $3.88 \mathrm{E}-07$ & -1.2446313 & 9 & 0.3 & & \\
\hline DHR6:63364001 & 6 & 63364001 & 63366000 & 2000 & 1 & 3.45E-06 & -1.5071319 & 6 & 0.3 & & \\
\hline DHR6:65071001 & 6 & 65071001 & 65072000 & 1000 & 1 & $5.52 \mathrm{E}-06$ & -1.2499216 & 2 & 0.2 & & \\
\hline DHR6:68065001 & 6 & 68065001 & 68068000 & 3000 & 1 & $2.01 \mathrm{E}-06$ & -1.3510918 & 6 & 0.2 & & \\
\hline DHR6:71468001 & 6 & 71468001 & 71470000 & 2000 & 1 & 1.61E-06 & -1.0795293 & 4 & 0.2 & & \\
\hline DHR6:90162001 & 6 & 90162001 & 90165000 & 3000 & 1 & 5.94E-07 & -1.3866614 & 20 & 0.667 & & \\
\hline DHR6:100345001 & 6 & 100345001 & 100346000 & 1000 & 1 & $7.23 \mathrm{E}-08$ & -1.4049174 & 6 & 0.6 & Fut8 & Metabolism \\
\hline DHR6:104538001 & 6 & 104538001 & 104539000 & 1000 & 1 & $6.62 \mathrm{E}-06$ & 0.9225193 & 11 & 1.1 & Susd6 & \\
\hline DHR6:118285001 & 6 & 118285001 & 118286000 & 1000 & 1 & $1.01 \mathrm{E}-06$ & -1.645431 & 4 & 0.4 & & \\
\hline DHR6:120978001 & 6 & 120978001 & 120980000 & 2000 & 1 & $3.38 \mathrm{E}-08$ & -1.676635 & 19 & 0.95 & & \\
\hline DHR6:124347001 & 6 & 124347001 & 124349000 & 2000 & 1 & $8.70 \mathrm{E}-06$ & 1.0558961 & 23 & 1.15 & Ttc7b & Metabolism \\
\hline DHR6:130724001 & 6 & 130724001 & 130726000 & 2000 & 1 & $4.08 \mathrm{E}-06$ & 0.9754726 & 25 & 1.25 & & \\
\hline DHR7:12270001 & 7 & 12270001 & 12272000 & 2000 & 1 & $2.35 \mathrm{E}-06$ & 1.0623791 & 62 & 3.1 & Apc2 & Cytoskeleton \\
\hline DHR7:12397001 & 7 & 12397001 & 12401000 & 4000 & 1 & 4.83E-06 & 0.873983 & 123 & 3.075 & Efna2;RGD1562114;Cirbp & Signaling;Transcription \\
\hline DHR7:13080001 & 7 & 13080001 & 13082000 & 2000 & 1 & 7.49E-06 & 1.0573686 & 30 & 1.5 & Plpp2 & \\
\hline DHR7:17778001 & 7 & 17778001 & 17780000 & 2000 & 1 & $3.16 \mathrm{E}-07$ & -1.4536079 & 8 & 0.4 & & \\
\hline DHR7:18113001 & 7 & 18113001 & 18114000 & 1000 & 1 & 9.03E-07 & -0.9674317 & 6 & 0.6 & Vom1r108 & \\
\hline DHR7:19687001 & 7 & 19687001 & 19688000 & 1000 & 1 & 1.39E-06 & -1.6283413 & 1 & 0.1 & & \\
\hline DHR7:21470001 & 7 & 21470001 & 21472000 & 2000 & 1 & $1.97 \mathrm{E}-06$ & -1.5232866 & 10 & 0.5 & & \\
\hline DHR7:27856001 & 7 & 27856001 & 27857000 & 1000 & 1 & $8.09 \mathrm{E}-06$ & -1.1388568 & 5 & 0.5 & & \\
\hline DHR7:36226001 & 7 & 36226001 & 36227000 & 1000 & 1 & $8.69 \mathrm{E}-06$ & 0.8919028 & 20 & 2 & & \\
\hline DHR7:43459001 & 7 & 43459001 & 43460000 & 1000 & 1 & $2.78 \mathrm{E}-07$ & -2.1734312 & 1 & 0.1 & & \\
\hline DHR7:52971001 & 7 & 52971001 & 52972000 & 1000 & 1 & $3.18 \mathrm{E}-06$ & 0.9862529 & 14 & 1.4 & & \\
\hline DHR7:53085001 & 7 & 53085001 & 53086000 & 1000 & 1 & 6.26E-06 & -1.0910849 & 8 & 0.8 & & \\
\hline DHR7:55960001 & 7 & 55960001 & 55963000 & 3000 & 1 & $8.53 \mathrm{E}-08$ & -1.8084681 & 16 & 0.533 & & \\
\hline DHR7:62342001 & 7 & 62342001 & 62343000 & 1000 & 1 & 7.30E-06 & -1.0893968 & 4 & 0.4 & AABR07057237.2 & \\
\hline DHR7:67847001 & 7 & 67847001 & 67848000 & 1000 & 1 & $2.88 \mathrm{E}-06$ & -1.0153564 & 1 & 0.1 & & \\
\hline DHR7:71180001 & 7 & 71180001 & 71181000 & 1000 & 1 & $3.23 \mathrm{E}-06$ & 0.8892696 & 10 & 1 & AABR07057436.1;Sdr9c7 & Metabolism \\
\hline DHR7:76260001 & 7 & 76260001 & 76261000 & 1000 & 1 & $2.76 \mathrm{E}-06$ & 0.9121376 & 15 & 1.5 & Ncald & Signaling \\
\hline DHR7:87780001 & 7 & 87780001 & 87781000 & 1000 & 1 & $8.86 \mathrm{E}-07$ & -1.1650355 & 7 & 0.7 & AABR07057765.1 & \\
\hline DHR7:97046001 & 7 & 97046001 & 97047000 & 1000 & 1 & 5.64E-06 & -1.1728706 & 5 & 0.5 & SIc22a 22 & \\
\hline DHR7:107716001 & 7 & 107716001 & 107717000 & 1000 & 1 & $9.21 \mathrm{E}-06$ & 0.9971403 & 16 & 1.6 & $\mathrm{Ccn} 4$ & \\
\hline DHR7:111676001 & 7 & 111676001 & 111679000 & 3000 & 1 & $1.83 \mathrm{E}-07$ & -1.4470555 & 8 & 0.267 & AABR07058360.3 & \\
\hline DHR7:112628001 & 7 & 112628001 & 112629000 & 1000 & 1 & 5.54E-06 & -1.2908773 & 6 & 0.6 & & \\
\hline DHR7:115530001 & 7 & 115530001 & 115532000 & 2000 & 1 & $4.85 \mathrm{E}-06$ & 0.8956183 & 18 & 0.9 & & \\
\hline DHR7:118424001 & 7 & 118424001 & 118425000 & 1000 & 1 & $2.78 \mathrm{E}-06$ & 1.0136008 & 20 & 2 & & \\
\hline DHR7:119475001 & 7 & 119475001 & 119477000 & 2000 & 1 & 6.15E-06 & 0.8515441 & 30 & 1.5 & Ncf4 & Development \\
\hline DHR7:122811001 & 7 & 122811001 & 122813000 & 2000 & 1 & $8.53 \mathrm{E}-06$ & 1.0733081 & 29 & 1.45 & Gm23880;AABR07058539.1 & \\
\hline DHR7:135477001 & 7 & 135477001 & 135480000 & 3000 & 1 & $9.13 \mathrm{E}-07$ & 1.072002 & 51 & 1.7 & Adamts20 & Protease \\
\hline DHR7:137802001 & 7 & 137802001 & 137803000 & 1000 & 1 & $2.09 \mathrm{E}-06$ & 0.8827294 & 16 & 1.6 & Arid2;AABR07058795.1;Scaf11 & \\
\hline DHR7:142472001 & 7 & 142472001 & 142476000 & 4000 & 1 & 9.05E-07 & 1.1053447 & 73 & 1.825 & SIc4a8 & Transport \\
\hline DHR7:145381001 & 7 & 145381001 & 145382000 & 1000 & 1 & $1.51 \mathrm{E}-06$ & 1.2141797 & 18 & 1.8 & AABR07058955.1;AABR07058955.2 & \\
\hline DHR8:1981001 & 8 & 1981001 & \begin{tabular}{|l|}
1983000 \\
\end{tabular} & 2000 & 1 & $1.98 \mathrm{E}-06$ & -1.3486421 & 4 & 0.2 & Gria4 & Signaling \\
\hline DHR8:2264001 & 8 & 2264001 & 2265000 & 1000 & 1 & 7.69E-06 & -1.3279399 & 0 & 0 & & \\
\hline DHR8:2753001 & 8 & 2753001 & 2755000 & 2000 & 1 & $9.35 \mathrm{E}-06$ & -1.4171475 & 3 & 0.15 & & \\
\hline DHR8:3625001 & 8 & 3625001 & 3628000 & 3000 & 1 & 6.47E-06 & -1.2266618 & 8 & 0.267 & & \\
\hline
\end{tabular}




\begin{tabular}{|c|c|c|c|c|c|c|c|c|c|c|c|}
\hline DHR8:3641001 & 8 & 3641001 & 3642000 & 1000 & 1 & \begin{tabular}{|l|}
$4.49 \mathrm{E}-07$ \\
\end{tabular} & -1.4286283 & 1 & 0.1 & Vom1r24 & \\
\hline DHR8:4193001 & 8 & 4193001 & 4194000 & 1000 & 1 & 3.09E-06 & -1.5222035 & 4 & 0.4 & & \\
\hline DHR8:5058001 & 8 & 5058001 & 5060000 & 2000 & 1 & $2.93 \mathrm{E}-06$ & -1.0610318 & 11 & 0.55 & & \\
\hline DHR8:11104001 & 8 & 11104001 & 11107000 & 3000 & 1 & $1.18 \mathrm{E}-07$ & -1.8200042 & 23 & 0.767 & & \\
\hline DHR8:15902001 & 8 & 15902001 & 15904000 & 2000 & 1 & $4.31 \mathrm{E}-06$ & -1.3676315 & 8 & 0.4 & & \\
\hline DHR8:16937001 & 8 & 16937001 & 16939000 & 2000 & 1 & \begin{tabular}{|l|}
$4.56 \mathrm{E}-06$ \\
\end{tabular} & $\begin{array}{l}-1.2195571 \\
\end{array}$ & 7 & 0.35 & & \\
\hline DHR8:19434001 & 8 & 19434001 & 19435000 & 1000 & 1 & \begin{tabular}{|l|}
$4.06 \mathrm{E}-07$ \\
\end{tabular} & -1.3157988 & 7 & 0.7 & & \\
\hline DHR8:25576001 & 8 & 25576001 & 25577000 & 1000 & 1 & 3.21E-06 & -1.1714675 & 6 & 0.6 & Dpy19l1 & Unknown \\
\hline DHR8:28559001 & 8 & 28559001 & 28561000 & 2000 & 1 & \begin{tabular}{|l|}
$4.27 \mathrm{E}-06$ \\
\end{tabular} & -1.2569489 & 16 & 0.8 & & \\
\hline DHR8:34409001 & 8 & 34409001 & 34410000 & 1000 & 1 & \begin{tabular}{|l|}
$4.10 \mathrm{E}-07$ \\
\end{tabular} & 0.9505369 & 20 & 2 & & \\
\hline DHR8:37818001 & 8 & 37818001 & 37819000 & 1000 & 1 & $3.02 \mathrm{E}-06$ & -1.1135347 & 1 & 0.1 & & \\
\hline DHR8:39706001 & 8 & 39706001 & 39709000 & 3000 & 1 & 9.67E-06 & 0.931665 & 46 & 1.533 & Tmem218;Slc37a2 & Metabolism \\
\hline DHR8:44441001 & 8 & 44441001 & 44442000 & 1000 & 1 & 7.44E-06 & 1.0217067 & 11 & 1.1 & & \\
\hline DHR8:44956001 & 8 & 44956001 & 44958000 & 2000 & 1 & $6.25 \mathrm{E}-07$ & 0.9778571 & 43 & 2.15 & Clmp & Receptor \\
\hline DHR8:58540001 & 8 & 58540001 & 58542000 & 2000 & 1 & $8.35 \mathrm{E}-08$ & 0.9211207 & 31 & 1.55 & Elmod1 & Signaling \\
\hline DHR8:61464001 & 8 & 61464001 & 61466000 & 2000 & 1 & $3.73 \mathrm{E}-08$ & 1.107157 & 27 & 1.35 & & \\
\hline DHR8:61528001 & 8 & 61528001 & 61529000 & 1000 & 1 & $1.26 \mathrm{E}-06$ & 1.1758572 & 12 & 1.2 & Odf3I1;Cspg4 & Cytoskeleton \\
\hline DHR8:61682001 & 8 & 61682001 & 61684000 & 2000 & 1 & $2.59 \mathrm{E}-06$ & -1.3232783 & 16 & 0.8 & Ptpn9 & Signaling \\
\hline DHR8:73282001 & 8 & 73282001 & 73283000 & 1000 & 1 & \begin{tabular}{|l|}
$7.17 \mathrm{E}-06$ \\
\end{tabular} & 1.0884246 & 16 & 1.6 & & \\
\hline DHR8:76429001 & 8 & 76429001 & 76432000 & 3000 & 1 & $6.48 \mathrm{E}-06$ & -1.2065422 & 24 & 0.8 & Bnip2;LOC103695118 & \\
\hline DHR8:77978001 & 8 & 77978001 & 77979000 & 1000 & 1 & 3.46E-06 & -0.9343226 & 4 & 0.4 & AC132740.1;Polr2m & \\
\hline DHR8:83942001 & 8 & 83942001 & 83944000 & 2000 & 1 & \begin{tabular}{|l|}
$6.34 \mathrm{E}-06$ \\
\end{tabular} & -1.2453229 & 11 & 0.55 & & \\
\hline DHR8:97913001 & 8 & 97913001 & 97914000 & 1000 & 1 & $1.93 \mathrm{E}-07$ & -1.3797465 & 5 & 0.5 & & \\
\hline DHR8:98307001 & 8 & 98307001 & 98308000 & 1000 & 1 & \begin{tabular}{|l|}
$9.79 \mathrm{E}-07$ \\
\end{tabular} & -1.5810798 & 0 & 0 & & \\
\hline DHR8:116459001 & 8 & 116459001 & 116464000 & 5000 & 1 & $6.98 \mathrm{E}-08$ & 1.141495 & 69 & 1.38 & Sema3f & Growth Factors \& Cytokines \\
\hline DHR8:116760001 & 8 & 116760001 & 116763000 & 3000 & 1 & 7.43E-06 & 1.1110377 & 49 & 1.633 & $\begin{array}{l}\text { Traip;Inka1;AC128059.3;Uba7;AC1280 } \\
59.5\end{array}$ & Signaling;Metabolism \\
\hline DHR8:127552001 & 8 & 127552001 & 127556000 & 4000 & 1 & 5.19E-06 & 1.0540764 & 51 & 1.275 & Itga9 & Extracellular Matrix \\
\hline DHR8:128739001 & 8 & 128739001 & 128743000 & 4000 & 1 & $8.82 \mathrm{E}-06$ & 1.0809658 & 101 & 2.525 & Cx3cr1;AABR07071742.3 & Growth Factors \& Cytokines \\
\hline DHR8:129049001 & 8 & 129049001 & 129051000 & 2000 & 1 & $8.38 \mathrm{E}-06$ & 1.0536607 & 40 & 2 & Myrip & Metabolism \\
\hline DHR9:34227001 & 9 & 34227001 & 34229000 & 2000 & 1 & $2.01 \mathrm{E}-06$ & -1.2111541 & 19 & 0.95 & & \\
\hline DHR9:35553001 & 9 & 35553001 & 35556000 & 3000 & 1 & $5.31 \mathrm{E}-06$ & -1.1280068 & 20 & 0.667 & & \\
\hline DHR9:40592001 & 9 & 40592001 & 40594000 & 2000 & 1 & $2.60 \mathrm{E}-06$ & -1.1681839 & 4 & 0.2 & & \\
\hline DHR9:51385001 & 9 & 51385001 & 51386000 & 1000 & 1 & 4.49E-06 & -1.1979433 & 8 & 0.8 & Gulp1 & Development \\
\hline DHR9:52285001 & 9 & 52285001 & 52286000 & 1000 & 1 & $2.64 \mathrm{E}-06$ & -1.1773243 & 0 & 0 & & \\
\hline DHR9:68487001 & 9 & 68487001 & 68490000 & 3000 & 1 & $3.32 \mathrm{E}-06$ & -1.5447784 & 12 & 0.4 & Pard3b & Cell Junction \\
\hline DHR9:76303001 & 9 & 76303001 & 76305000 & 2000 & 1 & $7.48 \mathrm{E}-06$ & -1.1697284 & 11 & 0.55 & & \\
\hline DHR9:79096001 & 9 & 79096001 & 79097000 & 1000 & 1 & 1.85E-07 & -1.4686089 & 4 & 0.4 & & \\
\hline DHR9:85957001 & 9 & 85957001 & 85958000 & 1000 & 1 & 4.15E-06 & 1.0306726 & 4 & 0.4 & & \\
\hline DHR9:102526001 & 9 & 102526001 & 102528000 & 2000 & 1 & 9.76E-06 & -1.2706717 & 3 & 0.15 & & \\
\hline DHR9:104069001 & 9 & 104069001 & 104072000 & 3000 & 1 & $8.49 \mathrm{E}-06$ & -1.4239741 & 16 & 0.533 & & \\
\hline DHR9:114699001 & 9 & 114699001 & 114700000 & 1000 & 1 & $1.46 \mathrm{E}-06$ & 0.9261265 & 16 & 1.6 & Rab12 & Signaling \\
\hline DHR9:117148001 & 9 & 117148001 & 117149000 & 1000 & 1 & $8.13 \mathrm{E}-06$ & 0.8530135 & 13 & 1.3 & & \\
\hline DHR9:120955001 & 9 & 120955001 & 120957000 & 2000 & 1 & $6.00 \mathrm{E}-06$ & -1.1404585 & 12 & 0.6 & & \\
\hline DHR10:3774001 & 10 & 3774001 & 3778000 & 4000 & 1 & 2.67E-06 & 0.8363746 & 55 & 1.375 & Cpped1 & Signaling \\
\hline DHR10:13747001 & 10 & 13747001 & 13750000 & 3000 & 1 & $9.74 \mathrm{E}-06$ & 1.0520673 & 52 & 1.733 & Abca3 & Receptor \\
\hline DHR10:16119001 & 10 & 16119001 & 16120000 & 1000 & 1 & $2.78 \mathrm{E}-08$ & 1.0493789 & 10 & 1 & & \\
\hline DHR10:18903001 & 10 & 18903001 & 18905000 & 2000 & 1 & $4.61 \mathrm{E}-07$ & 1.320565 & 24 & 1.2 & Kcnip1;Kcnmb1 & Signaling;Metabolism \\
\hline DHR10:23813001 & 10 & 23813001 & 23815000 & 2000 & 1 & $6.13 \mathrm{E}-07$ & 0.9743229 & 32 & 1.6 & Ebf1 & Transcription \\
\hline DHR10:38994001 & 10 & 38994001 & 38995000 & 1000 & 1 & 3.33E-06 & 0.9526139 & 15 & 1.5 & |l13;Rad50 & Signaling;Transcription \\
\hline DHR10:44145001 & 10 & 44145001 & 44147000 & 2000 & 1 & 6.51E-06 & -1.0739713 & 10 & 0.5 & Olr1424;AC097901.1;OIr1425 & Receptor \\
\hline DHR10:70613001 & 10 & 70613001 & 70614000 & 1000 & 1 & 4.79E-06 & 0.8817755 & 12 & 1.2 & Ap2b1 & Transport \\
\hline DHR10:74245001 & 10 & 74245001 & 74250000 & 5000 & 1 & $6.57 \mathrm{E}-06$ & 1.06488 & 85 & 1.7 & Ypel2 & \\
\hline DHR10:95098001 & 10 & 95098001 & 95099000 & 1000 & 1 & $5.21 \mathrm{E}-06$ & -1.0655352 & 7 & 0.7 & & \\
\hline DHR10:96277001 & 10 & 96277001 & 96279000 & 2000 & 1 & $1.48 \mathrm{E}-06$ & 0.9399712 & 49 & 2.45 & Prkca & Binding Protein \\
\hline DHR10:96750001 & 10 & 96750001 & 96752000 & 2000 & 1 & $3.70 \mathrm{E}-06$ & -0.7093502 & 15 & 0.75 & Cep112 & \\
\hline DHR10:97728001 & 10 & \begin{tabular}{|l}
97728001 \\
\end{tabular} & \begin{tabular}{|l|}
97729000 \\
\end{tabular} & 1000 & 1 & $5.28 \mathrm{E}-06$ & 1.1024783 & 20 & 2 & Amz2;SIc16a6 & \\
\hline DHR10:103221001 & 10 & 103221001 & 103223000 & 2000 & 1 & 6.41E-06 & 0.8737851 & 36 & 1.8 & Ttyh2 & Transport \\
\hline DHR10:103248001 & 10 & 103248001 & 103249000 & 1000 & 1 & $6.92 \mathrm{E}-06$ & 1.1009296 & 22 & 2.2 & Ttyh2 & Transport \\
\hline DHR10:105591001 & 10 & 105591001 & 105597000 & 6000 & 1 & $5.84 \mathrm{E}-06$ & 0.9834115 & 132 & 2.2 & Rhbdf2 & Protease \\
\hline DHR11:1859001 & 11 & 1859001 & 1862000 & 3000 & 1 & $3.44 \mathrm{E}-06$ & -1.2698788 & 15 & 0.5 & & \\
\hline DHR11:3165001 & 11 & 3165001 & 3167000 & 2000 & 1 & $2.41 \mathrm{E}-06$ & -1.0602401 & 16 & 0.8 & Vgll3 & Unknown \\
\hline DHR11:4768001 & 11 & 4768001 & 4771000 & 3000 & 1 & $5.30 \mathrm{E}-07$ & -1.3594324 & 10 & 0.333 & & \\
\hline DHR11:7834001 & 11 & 7834001 & 7835000 & 1000 & 1 & 4.32E-06 & -1.4921393 & 5 & 0.5 & & \\
\hline DHR11:8586001 & 11 & 8586001 & 8587000 & 1000 & 1 & $3.42 \mathrm{E}-06$ & -1.3950885 & 4 & 0.4 & & \\
\hline DHR11:11376001 & 11 & 11376001 & 11377000 & 1000 & 1 & $6.61 \mathrm{E}-06$ & -1.1774264 & 7 & 0.7 & Robo2 & Receptor \\
\hline DHR11:12131001 & 11 & 12131001 & 12132000 & 1000 & 1 & $9.05 \mathrm{E}-06$ & -1.0990746 & 2 & 0.2 & & \\
\hline DHR11:13130001 & 11 & 13130001 & 13132000 & 2000 & 1 & 1.45E-06 & -1.1535201 & 25 & 1.25 & & \\
\hline
\end{tabular}




\begin{tabular}{|c|c|c|c|c|c|c|c|c|c|c|c|}
\hline DHR11:21851001 & 11 & 21851001 & 21853000 & 2000 & 1 & $9.26 \mathrm{E}-06$ & \begin{tabular}{|l|}
-1.3657341 \\
\end{tabular} & 11 & 0.55 & & \\
\hline DHR11:21926001 & 11 & 21926001 & 21927000 & 1000 & 1 & $7.28 \mathrm{E}-06$ & -1.1817822 & 2 & 0.2 & & \\
\hline DHR11:23349001 & 11 & 23349001 & 23353000 & 4000 & 1 & $2.54 \mathrm{E}-06$ & -1.6608749 & 11 & 0.275 & & \\
\hline DHR11:25953001 & 11 & 25953001 & 25954000 & 1000 & 1 & $9.53 \mathrm{E}-06$ & -1.298161 & 1 & 0.1 & & \\
\hline DHR11:27518001 & 11 & 27518001 & 27519000 & 1000 & 1 & $3.76 \mathrm{E}-06$ & 1.0698225 & 12 & 1.2 & & \\
\hline DHR11:35103001 & 11 & 35103001 & 35108000 & 5000 & 1 & $6.97 \mathrm{E}-06$ & 0.946887 & 58 & 1.16 & Kcnj6 & Metabolism \\
\hline DHR11:36281001 & 11 & 36281001 & 36282000 & 1000 & 1 & $8.93 \mathrm{E}-06$ & 0.8529387 & 16 & 1.6 & & \\
\hline DHR11:39881001 & 11 & 39881001 & 39882000 & 1000 & 1 & $5.74 \mathrm{E}-06$ & -1.2866405 & 4 & 0.4 & & \\
\hline DHR11:43182001 & 11 & 43182001 & 43185000 & 3000 & 1 & $9.74 \mathrm{E}-06$ & -1.3016165 & 11 & 0.367 & Olr1531;Olr1532 & \\
\hline DHR11:48266001 & 11 & 48266001 & 48267000 & 1000 & 1 & $5.06 \mathrm{E}-06$ & -1.2612983 & 3 & 0.3 & & \\
\hline DHR11:51991001 & 11 & 51991001 & 51994000 & 3000 & 1 & $3.43 \mathrm{E}-07$ & -1.3555853 & 8 & 0.267 & & \\
\hline DHR11:57614001 & 11 & 57614001 & 57620000 & 6000 & 1 & $9.53 \mathrm{E}-06$ & -1.2765388 & 30 & 0.5 & & \\
\hline DHR11:63856001 & 11 & 63856001 & 63859000 & 3000 & 1 & $7.59 \mathrm{E}-06$ & -1.0671245 & 19 & 0.633 & & \\
\hline DHR11:65408001 & 11 & 65408001 & 65409000 & 1000 & 1 & $8.46 \mathrm{E}-06$ & -1.2197159 & 6 & 0.6 & & \\
\hline DHR11:69453001 & 11 & 69453001 & 69454000 & 1000 & 1 & $1.21 \mathrm{E}-06$ & 0.8411612 & 12 & 1.2 & & \\
\hline DHR11:70085001 & 11 & 70085001 & 70088000 & 3000 & 1 & $8.21 \mathrm{E}-07$ & 1.0067433 & 71 & 2.367 & Itgb5 & Receptor \\
\hline DHR11:73342001 & 11 & 73342001 & 73345000 & 3000 & 1 & $2.41 \mathrm{E}-06$ & 0.9930107 & 43 & 1.433 & & \\
\hline DHR11:73795001 & 11 & 73795001 & 73796000 & 1000 & 1 & $9.34 \mathrm{E}-06$ & 1.3840446 & 30 & 3 & & \\
\hline DHR11:75769001 & 11 & 75769001 & 75770000 & 1000 & 1 & $4.28 \mathrm{E}-06$ & -1.3308983 & 2 & 0.2 & & \\
\hline DHR11:78666001 & 11 & 78666001 & 78667000 & 1000 & 1 & $8.32 \mathrm{E}-07$ & 1.0927562 & 3 & 0.3 & & \\
\hline DHR11:85166001 & 11 & 85166001 & 85168000 & 2000 & 1 & $7.39 \mathrm{E}-07$ & -1.26914 & 5 & 0.25 & Olr1565 & \\
\hline DHR11:86940001 & 11 & 86940001 & 86944000 & 4000 & 1 & $9.78 \mathrm{E}-06$ & 1.0216835 & 64 & 1.6 & AABR07072264.4 & \\
\hline DHR12:1478001 & 12 & 1478001 & 1480000 & 2000 & 1 & $3.77 \mathrm{E}-06$ & -1.3781175 & 11 & 0.55 & $\mathrm{Rfc3}$ & Transcription \\
\hline DHR12:2282001 & 12 & 2282001 & 2283000 & 1000 & 1 & $7.54 \mathrm{E}-07$ & 0.9485395 & 11 & 1.1 & Clec4g & Receptor \\
\hline DHR12:9754001 & 12 & 9754001 & 9756000 & 2000 & 1 & $1.60 \mathrm{E}-06$ & 0.9587395 & 31 & 1.55 & $\operatorname{Ln} \times 2$ & Cytoskeleton \\
\hline DHR12:13573001 & 12 & 13573001 & 13574000 & 1000 & 1 & $2.90 \mathrm{E}-06$ & 0.9127797 & 8 & 0.8 & Rnf216 & Metabolism \\
\hline DHR12:18767001 & 12 & 18767001 & 18768000 & 1000 & 1 & $4.96 \mathrm{E}-06$ & -1.2056476 & 2 & 0.2 & & \\
\hline DHR12:19371001 & 12 & 19371001 & 19373000 & 2000 & 1 & $3.25 \mathrm{E}-06$ & \begin{tabular}{|l|}
-1.3843127 \\
\end{tabular} & 5 & 0.25 & & \\
\hline DHR12:22457001 & 12 & 22457001 & 22459000 & 2000 & 1 & $9.60 \mathrm{E}-07$ & 0.9827786 & 95 & 4.75 & SIc12a9;Trip6;AABR07035787.1 & Transport;Cytoskeleton \\
\hline DHR12:29500001 & 12 & 29500001 & 29502000 & 2000 & 1 & $6.69 \mathrm{E}-06$ & 0.9110661 & 23 & 1.15 & Caln1 & Signaling \\
\hline DHR12:40992001 & 12 & 40992001 & 40994000 & 2000 & 1 & $4.11 \mathrm{E}-06$ & 0.8717805 & 15 & 0.75 & & \\
\hline DHR12:42501001 & 12 & 42501001 & 42504000 & 3000 & 1 & $4.69 \mathrm{E}-06$ & 0.8855786 & 95 & 3.167 & Tbx3 & Epigenetic \\
\hline DHR13:2520001 & 13 & 2520001 & 2521000 & 1000 & 1 & $2.02 \mathrm{E}-06$ & $\mid-1.3797211$ & 1 & 0.1 & & \\
\hline DHR13:7906001 & 13 & 7906001 & 7911000 & 5000 & 1 & $2.27 \mathrm{E}-06$ & -1.7511888 & 19 & 0.38 & & \\
\hline DHR13:10093001 & 13 & 10093001 & 10094000 & 1000 & 1 & $5.15 \mathrm{E}-06$ & -1.2951725 & 2 & 0.2 & & \\
\hline DHR13:12223001 & 13 & 12223001 & 12226000 & 3000 & 1 & $1.95 \mathrm{E}-06$ & -1.331543 & 21 & 0.7 & & \\
\hline DHR13:12612001 & 13 & 12612001 & 12613000 & 1000 & 1 & $7.87 \mathrm{E}-06$ & -0.9102025 & 7 & 0.7 & & \\
\hline DHR13:19812001 & 13 & 19812001 & 19815000 & 3000 & 1 & $5.96 \mathrm{E}-06$ & -1.6686157 & 9 & 0.3 & & \\
\hline DHR13:20850001 & 13 & 20850001 & 20853000 & 3000 & 1 & $2.88 \mathrm{E}-07$ & -1.6772633 & 6 & 0.2 & & \\
\hline DHR13:21001001 & 13 & 21001001 & 21003000 & 2000 & 1 & $7.81 \mathrm{E}-06$ & -1.0797438 & 4 & 0.2 & & \\
\hline DHR13:22730001 & 13 & 22730001 & 22731000 & 1000 & 1 & $2.23 \mathrm{E}-08$ & -1.8714531 & 3 & 0.3 & & \\
\hline DHR13:299500001 & 13 & 29950001 & 29951000 & 1000 & 1 & $8.34 \mathrm{E}-07$ & -1.1938719 & 2 & 0.2 & & \\
\hline DHR13:31217001 & 13 & 31217001 & 31218000 & 1000 & 1 & $6.99 \mathrm{E}-06$ & -1.4923663 & 3 & 0.3 & Cdh7 & Extracellular Matrix \\
\hline DHR13:32306001 & 13 & 32306001 & 32307000 & 1000 & 1 & $7.98 \mathrm{E}-06$ & -1.0710778 & 3 & 0.3 & & \\
\hline DHR13:34914001 & 13 & 34914001 & 34918000 & 4000 & 1 & $7.64 \mathrm{E}-06$ & 1.1093931 & 44 & 1.1 & Gli2 & Transcription \\
\hline DHR13:35017001 & 13 & 35017001 & 35021000 & 4000 & 1 & $2.12 \mathrm{E}-06$ & 0.9350764 & 57 & 1.425 & Gli2 & Transcription \\
\hline DHR13:43143001 & 13 & 43143001 & 43145000 & 2000 & 1 & $9.90 \mathrm{E}-06$ & -1.4818426 & 13 & 0.65 & & \\
\hline DHR13:44873001 & 13 & 44873001 & 44876000 & 3000 & 1 & $6.84 \mathrm{E}-07$ & -1.4186253 & 12 & 0.4 & R3hdm1 & Unknown \\
\hline DHR13:47686001 & 13 & 47686001 & 47687000 & 1000 & 1 & $5.49 \mathrm{E}-06$ & -1.2089384 & 5 & 0.5 & & \\
\hline DHR13:56256001 & 13 & 56256001 & 56258000 & 2000 & 1 & 9.57E-06 & -1.0824012 & 13 & 0.65 & AABR07021086.1 & \\
\hline DHR13:57358001 & 13 & 57358001 & 57359000 & 1000 & 1 & $6.18 \mathrm{E}-06$ & -1.2551028 & 8 & 0.8 & Kcnt2 & Transport \\
\hline DHR13:58288001 & 13 & 58288001 & 58289000 & 1000 & 1 & $9.55 \mathrm{E}-06$ & \begin{tabular}{|l|}
-1.4240193 \\
\end{tabular} & 2 & 0.2 & & \\
\hline DHR13:59249001 & 13 & 59249001 & 59251000 & 2000 & 1 & $7.08 \mathrm{E}-06$ & -1.2867996 & 3 & 0.15 & & \\
\hline DHR13:63648001 & 13 & 63648001 & 63650000 & 2000 & 1 & $8.77 \mathrm{E}-06$ & -1.4769727 & 9 & 0.45 & Brinp3 & \\
\hline DHR13:65108001 & 13 & 65108001 & 65110000 & 2000 & 1 & $5.12 \mathrm{E}-08$ & -1.5210534 & 14 & 0.7 & & \\
\hline DHR13:66005001 & 13 & 66005001 & 66007000 & 2000 & 1 & $1.05 \mathrm{E}-06$ & -1.5845514 & 11 & 0.55 & & \\
\hline DHR13:73285001 & 13 & 73285001 & 73286000 & 1000 & 1 & $1.24 \mathrm{E}-06$ & 0.9241502 & 7 & 0.7 & Acbd6 & Metabolism \\
\hline DHR13:79755001 & 13 & 79755001 & 79757000 & 2000 & 1 & $2.83 \mathrm{E}-06$ & -1.4054128 & 10 & 0.5 & Suco & \\
\hline DHR13:100061001 & 13 & 100061001 & 100063000 & 2000 & 1 & $5.30 \mathrm{E}-06$ & 1.1165036 & 25 & 1.25 & AABR07021946.1;AABR07021946.2 & \\
\hline DHR13:111314001 & 13 & 111314001 & 111316000 & 2000 & 1 & $6.53 \mathrm{E}-06$ & 0.9723461 & 31 & 1.55 & Hhat & Metabolism \\
\hline DHR13:111933001 & 13 & 111933001 & 111935000 & 2000 & 1 & $8.63 \mathrm{E}-06$ & 1.0121967 & 17 & 0.85 & Hsd11b1 & Metabolism \\
\hline DHR13:113779001 & 13 & 113779001 & 113781000 & 2000 & 1 & $9.81 \mathrm{E}-07$ & 1.1527244 & 8 & 0.4 & Mir29b2;Mir3556b;Cd46 & Immune \\
\hline DHR14:4424001 & 14 & 4424001 & \begin{tabular}{|l|}
4425000 \\
\end{tabular} & 1000 & 1 & $2.90 \mathrm{E}-07$ & 0.958347 & 17 & 1.7 & & \\
\hline DHR14:22591001 & 14 & 22591001 & 22592000 & 1000 & 1 & $1.71 \mathrm{E}-06$ & -1.4736995 & 2 & 0.2 & Ugt2a3;Ugt2b35 & Metabolism \\
\hline DHR14:24574001 & 14 & 24574001 & 24576000 & 2000 & 1 & $5.51 \mathrm{E}-07$ & \begin{tabular}{|c|}
-1.0986763 \\
\end{tabular} & 8 & 0.4 & & \\
\hline DHR14:25472001 & 14 & 25472001 & 25473000 & 1000 & 1 & $7.88 \mathrm{E}-06$ & -1.5045223 & 2 & 0.2 & & \\
\hline DHR14:26691001 & 14 & 26691001 & 26693000 & 2000 & 1 & $1.36 \mathrm{E}-06$ & -1.3513566 & 11 & 0.55 & Tecrl & \\
\hline DHR14:26851001 & 14 & 26851001 & 26852000 & 1000 & 1 & $9.52 \mathrm{E}-06$ & -1.4436006 & 6 & 0.6 & & \\
\hline DHR14:27644001 & 14 & 27644001 & 27646000 & 2000 & 1 & $8.77 \mathrm{E}-06$ & -1.8126304 & 7 & 0.35 & & \\
\hline
\end{tabular}




\begin{tabular}{|c|c|c|c|c|c|c|c|c|c|c|c|}
\hline DHR14:29348001 & 14 & 29348001 & 29350000 & 2000 & 1 & \begin{tabular}{|l|}
$7.28 \mathrm{E}-06$ \\
\end{tabular} & -1.2694122 & 8 & 0.4 & & \\
\hline DHR14:31023001 & 14 & 31023001 & 31024000 & 1000 & 1 & $2.39 \mathrm{E}-07$ & -1.3872805 & 3 & 0.3 & AABR07014769.1 & \\
\hline DHR14:32964001 & 14 & 32964001 & 32966000 & 2000 & 1 & $8.03 \mathrm{E}-06$ & 0.8081918 & 25 & 1.25 & & \\
\hline DHR14:39392001 & 14 & 39392001 & 39394000 & 2000 & 1 & $1.61 \mathrm{E}-06$ & 0.8765383 & 61 & 3.05 & & \\
\hline DHR14:47178001 & 14 & 47178001 & 47180000 & 2000 & 1 & 4.79E-06 & -1.291933 & 15 & 0.75 & & \\
\hline DHR14:48619001 & 14 & 48619001 & 48620000 & 1000 & 1 & 4.77E-06 & -0.6450885 & 14 & $\begin{array}{ll}1.4 \\
\end{array}$ & Dthd1 & \\
\hline DHR14:49049001 & 14 & 49049001 & 49053000 & 4000 & 1 & 6.56E-06 & -1.2304093 & 8 & 0.2 & & \\
\hline DHR14:52045001 & 14 & 52045001 & 52049000 & 4000 & 1 & $5.71 \mathrm{E}-06$ & \begin{tabular}{|l|}
-1.3792903 \\
\end{tabular} & 11 & 0.275 & & \\
\hline \begin{tabular}{|l|} 
DHR14:52375001 \\
\end{tabular} & 14 & 52375001 & 52376000 & 1000 & 1 & 1.97E-06 & -1.7502305 & 2 & 0.2 & & \\
\hline DHR14:54599001 & 14 & 54599001 & 54605000 & 6000 & 1 & $7.20 \mathrm{E}-07$ & -1.5670502 & 38 & 0.633 & & \\
\hline DHR14:56584001 & 14 & 56584001 & 56586000 & 2000 & 1 & $6.28 \mathrm{E}-06$ & \begin{tabular}{|c|}
-1.2028879 \\
\end{tabular} & 8 & 0.4 & & \\
\hline DHR14:57463001 & 14 & 57463001 & 57466000 & 3000 & 1 & 6.17E-06 & -1.3248159 & 8 & 0.267 & & \\
\hline DHR14:57637001 & 14 & 57637001 & 57638000 & 1000 & 1 & $1.90 \mathrm{E}-06$ & -1.1496588 & 2 & 0.2 & & \\
\hline DHR14:63669001 & 14 & 63669001 & 63672000 & 3000 & 1 & 3.30E-07 & -1.2972214 & 8 & 0.267 & & \\
\hline DHR14:64578001 & 14 & 64578001 & 64579000 & 1000 & 1 & $2.51 \mathrm{E}-06$ & 0.8466982 & 6 & 0.6 & & \\
\hline DHR14:75447001 & 14 & 75447001 & 75450000 & 3000 & 1 & $1.25 \mathrm{E}-06$ & -1.545532 & 18 & 0.6 & & \\
\hline DHR14:80862001 & 14 & 80862001 & 80864000 & 2000 & 1 & $9.32 \mathrm{E}-06$ & 0.8842388 & 27 & 1.35 & & \\
\hline DHR14:82915001 & 14 & 82915001 & 82917000 & 2000 & 1 & $4.08 \mathrm{E}-07$ & 1.0091332 & 43 & 2.15 & SIc5a1 & Transport \\
\hline DHR14:85392001 & 14 & 85392001 & 85394000 & 2000 & 1 & $1.54 \mathrm{E}-06$ & 1.1746355 & 62 & 3.1 & Emid1 & Extracellular Matrix \\
\hline DHR14:88949001 & 14 & 88949001 & 88952000 & 3000 & 1 & $9.04 \mathrm{E}-06$ & 0.9337025 & 54 & 1.8 & & \\
\hline DHR14:90249001 & 14 & 90249001 & 90250000 & 1000 & 1 & $3.39 \mathrm{E}-06$ & -1.3797414 & 2 & 0.2 & & \\
\hline DHR14:94036001 & 14 & 94036001 & 94038000 & 2000 & 1 & $5.25 \mathrm{E}-06$ & -1.5711101 & 8 & 0.4 & & \\
\hline DHR14:96938001 & 14 & 96938001 & 96939000 & 1000 & 1 & $1.99 \mathrm{E}-06$ & -0.8540288 & 2 & 0.2 & & \\
\hline DHR14:112286001 & 14 & 112286001 & 112289000 & 3000 & 1 & $2.05 \mathrm{E}-07$ & -1.47181 & 14 & 0.467 & RNaseP_nuc & \\
\hline DHR15:7193001 & 15 & \begin{tabular}{|l|}
7193001 \\
\end{tabular} & \begin{tabular}{|l|}
7194000 \\
\end{tabular} & 1000 & 1 & $2.90 \mathrm{E}-07$ & -1.5539227 & 6 & 0.6 & & \\
\hline DHR15:7373001 & 15 & 7373001 & 7375000 & 2000 & 1 & 6.46E-06 & -1.1059301 & 7 & 0.35 & & \\
\hline DHR15:24955001 & 15 & 24955001 & 24957000 & 2000 & 1 & $9.00 \mathrm{E}-06$ & 0.8506991 & 44 & 2.2 & Peli2 & Signaling \\
\hline DHR15:30204001 & 15 & 30204001 & 30209000 & 5000 & 1 & $3.84 \mathrm{E}-06$ & -1.6711343 & 21 & 0.42 & AABR07017701.1 & \\
\hline DHR15:38415001 & 15 & 38415001 & 38416000 & 1000 & 1 & $3.71 \mathrm{E}-06$ & 0.9527257 & 13 & 1.3 & & \\
\hline DHR15:39838001 & 15 & 39838001 & 39839000 & 1000 & 1 & $1.66 \mathrm{E}-07$ & 0.9781521 & 13 & 1.3 & Cab39| & Signaling \\
\hline DHR15:46243001 & 15 & 46243001 & 46246000 & 3000 & 1 & $2.74 \mathrm{E}-06$ & 0.9841786 & 27 & 0.9 & Defb44 & \\
\hline DHR15:64065001 & 15 & 64065001 & 64066000 & 1000 & 1 & $3.50 \mathrm{E}-06$ & -1.3559519 & 2 & 0.2 & & \\
\hline DHR15:65111001 & 15 & 65111001 & 65113000 & 2000 & 1 & $2.17 \mathrm{E}-06$ & -1.2305104 & 9 & 0.45 & & \\
\hline DHR15:65552001 & 15 & 65552001 & 65556000 & 4000 & 1 & \begin{tabular}{|l|}
$9.80 \mathrm{E}-07$ \\
\end{tabular} & -1.3903227 & 20 & 0.5 & & \\
\hline DHR15:65911001 & 15 & 65911001 & 65913000 & 2000 & 1 & $1.90 \mathrm{E}-06$ & -1.403175 & 12 & 0.6 & & \\
\hline DHR15:66212001 & 15 & 66212001 & 66214000 & 2000 & 1 & 1.54E-06 & -1.5512106 & 6 & 0.3 & & \\
\hline DHR15:78297001 & 15 & 78297001 & 78298000 & 1000 & 1 & $4.06 \mathrm{E}-08$ & -1.7186372 & 4 & 0.4 & & \\
\hline DHR15:79362001 & 15 & 79362001 & 79363000 & 1000 & 1 & $2.01 \mathrm{E}-06$ & -1.5307378 & 7 & 0.7 & & \\
\hline DHR15:84287001 & 15 & 84287001 & 84288000 & 1000 & 1 & 9.53E-06 & -1.0644938 & 2 & 0.2 & & \\
\hline DHR15:91474001 & 15 & 91474001 & 91475000 & 1000 & 1 & $1.90 \mathrm{E}-06$ & -1.3982919 & 3 & 0.3 & Mycbp2;AABR07019155.1 & Metabolism \\
\hline DHR15:94310001 & 15 & 94310001 & 94312000 & 2000 & 1 & 6.41E-06 & -1.4070592 & 13 & 0.65 & & \\
\hline DHR15:94984001 & 15 & 94984001 & 94986000 & 2000 & 1 & 9.89E-06 & -1.372557 & 6 & 0.3 & & \\
\hline DHR15:96237001 & 15 & 96237001 & 96239000 & 2000 & 1 & 2.19E-09 & -1.6712154 & 11 & 0.55 & & \\
\hline DHR15:98231001 & 15 & 98231001 & 98233000 & 2000 & 1 & $3.36 \mathrm{E}-07$ & -1.6757572 & 7 & 0.35 & & \\
\hline DHR15:107067001 & 15 & 107067001 & 107069000 & 2000 & 1 & $8.26 \mathrm{E}-06$ & -1.2807314 & 13 & 0.65 & & \\
\hline DHR16:131001 & 16 & 131001 & 133000 & 2000 & 1 & $2.54 \mathrm{E}-09$ & -1.2544404 & 17 & 0.85 & AABR07024442.1 & \\
\hline DHR16:1508001 & 16 & 1508001 & 1511000 & 3000 & 1 & $5.80 \mathrm{E}-06$ & 0.8266503 & 49 & 1.633 & & \\
\hline DHR16:7130001 & 16 & 7130001 & 7131000 & 1000 & 1 & \begin{tabular}{|l|}
$8.77 E-07$ \\
\end{tabular} & -1.2203231 & 10 & 1 & Pbrm1 & Unknown \\
\hline DHR16:12967001 & 16 & 12967001 & 12971000 & 4000 & 1 & $1.78 \mathrm{E}-06$ & -1.0816536 & 24 & 0.6 & AABR07024732.1;AABR07024733.1 & \\
\hline DHR16:17132001 & 16 & 17132001 & 17133000 & 1000 & 1 & $5.56 \mathrm{E}-06$ & -1.2146039 & 3 & 0.3 & AABR07024799.1 & \\
\hline DHR16:27299001 & 16 & 27299001 & 27300000 & 1000 & 1 & $2.40 \mathrm{E}-07$ & -1.3403277 & 2 & 0.2 & & \\
\hline DHR16:41030001 & 16 & 41030001 & 41031000 & 1000 & 1 & $7.96 \mathrm{E}-07$ & -1.6532517 & 10 & 1 & AABR07025594.1 & \\
\hline DHR16:43766001 & 16 & 43766001 & 43767000 & 1000 & 1 & $7.83 \mathrm{E}-07$ & -1.3633368 & 5 & 0.5 & & \\
\hline DHR16:44605001 & 16 & 44605001 & 44607000 & 2000 & 1 & $6.52 \mathrm{E}-06$ & -1.3529863 & 5 & 0.25 & & \\
\hline DHR16:52546001 & 16 & 52546001 & 52550000 & 4000 & 1 & $3.62 \mathrm{E}-08$ & -1.658517 & 24 & 0.6 & U6 & \\
\hline DHR16:53415001 & 16 & 53415001 & 53417000 & 2000 & 1 & $4.35 \mathrm{E}-07$ & -1.5835313 & 7 & 0.35 & & \\
\hline DHR16:57180001 & 16 & 57180001 & 57182000 & 2000 & 1 & $6.84 \mathrm{E}-06$ & -1.8719072 & 25 & 1.25 & & \\
\hline DHR16:61298001 & 16 & 61298001 & 61299000 & 1000 & 1 & $1.80 \mathrm{E}-06$ & 1.0854358 & 7 & $\begin{array}{l}0.7 \\
\end{array}$ & & \\
\hline DHR16:72053001 & 16 & 72053001 & 72055000 & 2000 & 1 & $1.41 \mathrm{E}-07$ & -0.8018306 & 22 & 1.1 & Adam5 & \\
\hline DHR16:74280001 & 16 & 74280001 & 74281000 & 1000 & 1 & $2.79 \mathrm{E}-06$ & 0.8338042 & 27 & 2.7 & & \\
\hline DHR16:74871001 & 16 & 74871001 & 74873000 & 2000 & 1 & $9.08 \mathrm{E}-06$ & 0.9061835 & 19 & 0.95 & Alg11;Atp7b & Metabolism;Transport \\
\hline DHR16:81268001 & 16 & 81268001 & 81269000 & 1000 & 1 & 9.57E-06 & -1.2896122 & 4 & 0.4 & AABR07026534.3 & \\
\hline DHR16:83414001 & 16 & 83414001 & 83415000 & 1000 & 1 & $9.13 \mathrm{E}-06$ & 1.3607709 & 31 & 3.1 & Col4a2 & Cytoskeleton \\
\hline DHR17:28911001 & 17 & 28911001 & 28912000 & 1000 & 1 & $6.43 \mathrm{E}-06$ & -0.8596984 & 7 & 0.7 & & \\
\hline DHR17:29273001 & 17 & 29273001 & 29274000 & 1000 & 1 & 3.04E-06 & -1.1358825 & 6 & 0.6 & Fars2 & \\
\hline DHR17:34716001 & 17 & 34716001 & 34717000 & 1000 & 1 & $4.88 \mathrm{E}-06$ & 1.2128401 & 6 & 0.6 & Exoc2;AABR07027502.1 & \\
\hline DHR17:35823001 & 17 & 35823001 & 35824000 & 1000 & 1 & $9.88 \mathrm{E}-06$ & 0.9444305 & 13 & 1.3 & & \\
\hline DHR17:36613001 & 17 & 36613001 & 36614000 & 1000 & 1 & $4.30 \mathrm{E}-06$ & 0.9901922 & 12 & 1.2 & AABR07027567.1 & \\
\hline DHR17:40369001 & 17 & 40369001 & 40370000 & 1000 & 1 & $7.31 \mathrm{E}-06$ & -0.993551 & 5 & 0.5 & & \\
\hline
\end{tabular}




\begin{tabular}{|c|c|c|c|c|c|c|c|c|c|c|c|}
\hline DHR17:44881001 & 17 & 44881001 & 44884000 & 3000 & 1 & $9.63 \mathrm{E}-06$ & -1.3681324 & 3 & 0.1 & & \\
\hline DHR17:48814001 & 17 & 48814001 & 48815000 & 1000 & 1 & $6.19 \mathrm{E}-07$ & \begin{tabular}{|l|}
-1.3693727 \\
\end{tabular} & 7 & 0.7 & Vps41 & Transport \\
\hline DHR17:49124001 & 17 & 49124001 & 49125000 & 1000 & 1 & $2.74 \mathrm{E}-06$ & -1.1054878 & 4 & 0.4 & & \\
\hline DHR17:50888001 & 17 & 50888001 & 50890000 & 2000 & 1 & 9.61E-06 & \begin{tabular}{|l|}
-1.1863904 \\
\end{tabular} & 8 & 0.4 & & \\
\hline DHR17:64730001 & 17 & 64730001 & 64732000 & 2000 & 1 & $3.41 \mathrm{E}-06$ & -1.4303614 & 10 & 0.5 & & \\
\hline DHR17:69672001 & 17 & 69672001 & 69674000 & 2000 & 1 & \begin{tabular}{|l|}
$2.67 \mathrm{E}-06$ \\
\end{tabular} & -1.3707456 & 9 & 0.45 & Akr1c19 & Metabolism \\
\hline DHR17:70431001 & 17 & 70431001 & 70432000 & 1000 & 1 & $1.42 \mathrm{E}-06$ & 0.9975988 & 13 & 1.3 & Fbh1 & \\
\hline DHR17:71730001 & 17 & 71730001 & 71731000 & 1000 & 1 & $5.28 \mathrm{E}-06$ & 1.0894335 & 21 & 2.1 & Sfmbt2 & Transcription \\
\hline DHR18:1286001 & 18 & 1286001 & 1290000 & 4000 & 1 & 3.39E-06 & -1.4337112 & 13 & 0.325 & Rock1 & Signaling \\
\hline DHR18:2710001 & 18 & 2710001 & 2712000 & 2000 & 1 & \begin{tabular}{|l|}
$4.13 \mathrm{E}-07$ \\
\end{tabular} & 1.1115527 & 32 & 1.6 & & \\
\hline DHR18:34447001 & 18 & 34447001 & 34449000 & 2000 & 1 & $6.93 \mathrm{E}-06$ & -1.3169565 & 4 & 0.2 & & \\
\hline DHR18:39097001 & 18 & 39097001 & 39098000 & 1000 & 1 & 4.47E-06 & -1.2548324 & 1 & 0.1 & & \\
\hline DHR18:53079001 & 18 & 53079001 & 53081000 & 2000 & 1 & $9.03 \mathrm{E}-06$ & -1.0545386 & 38 & 1.9 & Fbn2;AC104053.2 & Extracellular Matrix \\
\hline DHR18:70342001 & 18 & 70342001 & 70345000 & 3000 & 1 & $1.12 \mathrm{E}-06$ & 1.0342285 & 50 & 1.667 & & \\
\hline DHR18:79735001 & 18 & 79735001 & 79738000 & 3000 & 1 & $2.20 \mathrm{E}-06$ & 1.0033043 & 60 & 2 & & \\
\hline DHR18:84897001 & 18 & 84897001 & 84898000 & 1000 & 1 & $4.72 \mathrm{E}-06$ & -1.4729389 & 1 & 0.1 & & \\
\hline DHR19:7079001 & 19 & 7079001 & 7081000 & 2000 & 1 & \begin{tabular}{|l|}
$1.73 \mathrm{E}-07$ \\
\end{tabular} & -1.5043608 & 8 & 0.4 & & \\
\hline DHR19:9587001 & 19 & 9587001 & 9589000 & 2000 & 1 & $9.75 \mathrm{E}-06$ & 0.9967526 & 99 & 4.95 & Got2 & Metabolism \\
\hline DHR19:11568001 & 19 & 11568001 & 11569000 & 1000 & 1 & $3.81 \mathrm{E}-06$ & 0.973311 & 10 & 1 & Gnao1 & Signaling \\
\hline DHR19:27537001 & 19 & 27537001 & 27540000 & 3000 & 1 & $6.99 \mathrm{E}-06$ & -1.072661 & 15 & 0.5 & Olr1666 & Receptor \\
\hline DHR19:37109001 & 19 & 37109001 & 37111000 & 2000 & 1 & $1.36 \mathrm{E}-06$ & 0.9437954 & 31 & 1.55 & Ces4a;U6 & \\
\hline DHR19:50122001 & 19 & 50122001 & 50126000 & 4000 & 1 & \begin{tabular}{|l|}
$7.54 \mathrm{E}-06$ \\
\end{tabular} & 0.8122062 & 57 & 1.425 & Plcg2 & Signaling \\
\hline DHR19:53081001 & 19 & 53081001 & 53084000 & 3000 & 1 & 4.47E-06 & 0.8885451 & 33 & 1.1 & & \\
\hline DHR19:54196001 & 19 & 54196001 & 54197000 & 1000 & 1 & $8.63 \mathrm{E}-06$ & 1.1943494 & 18 & 1.8 & RGD1309651 & \\
\hline DHR19:55354001 & 19 & 55354001 & 55355000 & 1000 & 1 & $6.75 \mathrm{E}-06$ & 0.8562963 & 16 & 1.6 & Piezo1 & \\
\hline DHR19:57403001 & 19 & 57403001 & 57404000 & 1000 & 1 & $4.62 \mathrm{E}-06$ & 0.9609608 & 16 & 1.6 & RGD1559896 & EST \\
\hline DHR19:58214001 & 19 & 58214001 & 58216000 & 2000 & 1 & 4.06E-06 & 1.0545314 & 34 & 1.7 & & \\
\hline DHR19:58524001 & 19 & 58524001 & 58525000 & 1000 & 1 & $2.67 \mathrm{E}-07$ & 1.0326376 & 17 & 1.7 & & \\
\hline DHR19:60645001 & 19 & 60645001 & 60647000 & 2000 & 1 & $3.28 \mathrm{E}-06$ & 0.8242639 & 27 & 1.35 & & \\
\hline DHR20:3288001 & 20 & 3288001 & 3291000 & 3000 & 1 & $8.12 \mathrm{E}-06$ & 0.9292302 & 41 & 1.367 & RT1-T24-1;Gnl1;Prr3 & Immune;Transcription \\
\hline DHR20:4986001 & 20 & 4986001 & 4987000 & 1000 & 1 & $9.29 \mathrm{E}-06$ & 0.9453185 & 22 & 2.2 & AABR07044408.1;AC094348.4;Vars & Metabolism \\
\hline DHR20:6185001 & 20 & 6185001 & 6188000 & 3000 & 1 & $9.88 \mathrm{E}-06$ & 0.9949027 & 60 & 2 & AABR07044444.2;Pxt1 & \\
\hline DHR20:24894001 & 20 & 24894001 & 24896000 & 2000 & 1 & $8.20 \mathrm{E}-07$ & -1.3772935 & 13 & 0.65 & & \\
\hline DHR20:26056001 & 20 & 26056001 & 26057000 & 1000 & 1 & $4.32 \mathrm{E}-06$ & -1.4434983 & 9 & 0.9 & Lrrtm3 & Receptor \\
\hline DHR20:29728001 & 20 & 29728001 & 29731000 & 3000 & 1 & $1.88 \mathrm{E}-06$ & 1.0959688 & 38 & 1.267 & Chst3 & Metabolism \\
\hline DHR20:30157001 & 20 & 30157001 & 30160000 & 3000 & 1 & $7.94 \mathrm{E}-06$ & 1.098396 & 54 & 1.8 & AABR07044980.1 & \\
\hline DHR20:36885001 & 20 & 36885001 & 36887000 & 2000 & 1 & $8.72 \mathrm{E}-06$ & -1.1624751 & 12 & 0.6 & & \\
\hline DHR20:37257001 & 20 & 37257001 & 37259000 & 2000 & 1 & 5.75E-06 & -1.4031875 & 2 & 0.1 & & \\
\hline DHR20:38832001 & 20 & 38832001 & 38837000 & 5000 & 1 & 5.49E-07 & -1.3738942 & 31 & 0.62 & & \\
\hline DHR20:42182001 & 20 & 42182001 & 42183000 & 1000 & 1 & $1.53 \mathrm{E}-06$ & -1.4339413 & 5 & 0.5 & & \\
\hline DHR20:42943001 & 20 & 42943001 & 42944000 & 1000 & 1 & $3.53 \mathrm{E}-06$ & -1.0960825 & 5 & 0.5 & & \\
\hline DHR20:53813001 & 20 & 53813001 & 53815000 & 2000 & 1 & 6.04E-06 & -1.4613786 & 10 & 0.5 & Grik2 & Signaling \\
\hline DHR20:53994001 & 20 & 53994001 & 53995000 & 1000 & 1 & $2.78 \mathrm{E}-06$ & -1.5229499 & 3 & 0.3 & Grik2 & Signaling \\
\hline DHR20:55909001 & 20 & 55909001 & 55911000 & 2000 & 1 & $3.91 \mathrm{E}-06$ & -1.6302831 & 8 & 0.4 & & \\
\hline DHRX:6852001 & $x$ & 6852001 & 6855000 & 3000 & 1 & 6.07E-08 & -0.8809166 & 29 & 0.967 & AABR07073516.1 & \\
\hline DHRX:15190001 & $x$ & 15190001 & 15191000 & 1000 & 1 & $8.86 \mathrm{E}-07$ & 1.3273143 & 6 & 0.6 & Suv39h1l1 & \\
\hline DHRX:16604001 & $\mathrm{x}$ & 16604001 & 16605000 & 1000 & 1 & 6.37E-06 & -1.5706523 & 8 & 0.8 & & \\
\hline DHRX:59070001 & $\mathrm{X}$ & 59070001 & 59071000 & 1000 & 1 & $5.28 \mathrm{E}-08$ & -1.8791795 & 6 & 0.6 & & \\
\hline DHRX:63816001 & $x$ & 63816001 & 63817000 & 1000 & 1 & $2.74 \mathrm{E}-06$ & -1.8626656 & 11 & 1.1 & $\begin{array}{l}\text { Maged1;AABR07038902.1;AABR07038 } \\
902.2\end{array}$ & Extracellular Matrix \\
\hline DHRX:92709001 & $x$ & 92709001 & 92710000 & 1000 & 1 & 7.61E-06 & -1.5913318 & 8 & 0.8 & & \\
\hline DHRX:103131001 & $x$ & 103131001 & 103132000 & 1000 & 1 & \begin{tabular}{|l|}
$3.46 \mathrm{E}-07$ \\
\end{tabular} & -2.167639 & 3 & 0.3 & & \\
\hline DHRX:106356001 & $x$ & 106356001 & 106358000 & 2000 & 1 & $2.60 \mathrm{E}-06$ & 1.5867972 & 47 & 2.35 & Gprasp2 & \\
\hline DHRX:125863001 & $\mathrm{X}$ & 125863001 & 125864000 & 1000 & 1 & \begin{tabular}{|l|}
$4.65 \mathrm{E}-06$ \\
\end{tabular} & -1.9180939 & 5 & 0.5 & & \\
\hline DHRX:127279001 & $x$ & 127279001 & 127282000 & 3000 & 1 & $9.40 \mathrm{E}-06$ & -1.5807267 & 14 & 0.467 & & \\
\hline DHRX:132474001 & $x$ & 132474001 & 132475000 & 1000 & 1 & $2.46 \mathrm{E}-06$ & -1.9711229 & 2 & 0.2 & & \\
\hline DHRX:152609001 & $x$ & 152609001 & 152610000 & 1000 & 1 & $4.42 \mathrm{E}-06$ & \begin{tabular}{|c|}
-1.7690737 \\
\end{tabular} & 3 & 0.3 & Gabra3 & Receptor \\
\hline
\end{tabular}

